\title{
Reassessing the origin of Triton
}

\author{
E. Nogueira \\ Observatorio Nacional/MCT, Rua General José Cristino 77; CEP 20921-400, Rio de Janeiro, RJ, Brasil
}

R. Brasser*

Dep. Cassiopée, University of Nice - Sophia Antipolis, CNRS, Observatoire de la Côte d'Azur; F-06304, Nice, France

R. Gomes

Observatorio Nacional/MCT, Rua General José Cristino 77; CEP 20921-400, Rio de Janeiro, RJ, Brasil

\section{Abstract}

The origin of Neptune's large, circular but retrograde satellite Triton has remained largely unexplained. There is an apparent 'consensus that its origin lies in it being captured, but until recently no successful capture mechanism has been found. Agnor \& Hamilton (2006) demonstrated that the disruption of a trans-Neptunian binary object which had Triton as a member, and which underwent a very close encounter with Neptune, was an effective mechanism to capture Triton while its former partner continued 'on a hyperbolic orbit. The subsequent evolution of Triton's post-capture orbit to its current one could have proceeded through 'gravitational tides (Correia, 2009), during which time Triton was most likely semi-molten (McKinnon, 1984). However, to date, no study has been performed that considered both the capture and the subsequent tidal evolution. Thus it is attempted here with the use of numerical simulations. The study by Agnor \& Hamilton (2006) is repeated in the framework of the Nice model (Tsiganis et al., 2005) to determine the post-capture orbit of Triton. After capture Triton is then subjected to tidal evolution using the model of Mignard (1979, 1980). The perturbations from the Sun and the figure of Neptune are included. The perturbations from the Sun acting on Triton just after its capture cause it to spend a long time in its high-eccentricity phase, usually of the order of $10 \mathrm{Myr}$ 'while the typical time to circularise to its current orbit is some $200 \mathrm{Myr}$, consistent with earlier studies. The current orbit of Triton is consistent with an origin through binary capture and tidal evolution, even though the model prefers Triton to be closer to Neptune than it is today. The probability of capturing Triton in this manner is approximately $0.7 \%$. Since the capture of Triton was at most a $50 \%$ event - since only Neptune has one, but Uranus does not - we deduce that in the primordial trans-Neptunian disc there were some 100 binaries with at least one Triton-sized member. Morbidelli et al. (2009) concludes there were some 1000 Triton-sized bodies in the trans-Neptunian proto-planetary disc, so the primordial binary fraction with at least one Triton-sized member is $10 \%$. This value is consistent with theoretical predictions, but at the low end. If Triton was captured at the same time as Neptune's irregular satellites, the far majority of these, including Nereid, would be lost. This suggests either that Triton was captured on an orbit with a small semi-major axis $a \lesssim 50 R_{N}$ (a rare event), or that it was captured before the dynamical instability of the Nice 'model, or that some other mechanism was at play. The issue of keeping the irregular satellites remains unresolved.

Keywords: Triton; Neptune, satellites; Tides, solid body; Satellites, dynamics; Irregular satellites

\section{Introduction and background}

Of all the large natural satellites of the planets, Neptune's Triton is one of the most peculiar. It orbits Neptune at a distance of $354759 \mathrm{~km}$ or $14.3 \mathrm{Neptune}$ radii $\left(R_{N}\right)$, with a period of 5.877 days (Jacobson et al., 1991), similar to some of Uranus' regular satellites. Its orbit is circular $\left(e \sim 10^{-5}\right)$ (Jacobson et al., 1991), yet its inclination with respect to Neptune's equator is $156.8^{\circ}$, thus retrograde and ruling out a formation from within the Neptune system. Its retrograde orbit has led to the belief that Triton was captured by Neptune from a heliocentric orbit (McKinnon, 1984; Benner \& McKinnon, 1995), and its Neptune-centric orbit subsequently decayed through either tidal interaction (McCord, 1966; McKinnon, 1984; Goldreich et al., 1989; Correia, 2009), Neptune's circumplanetary gas disc (McKinnon \& Leith, 1995) or a debris disc that formed from collisions among Neptune's putative regular satellites (Ćuk \& Gladman, 2005). We shall briefly discuss each of these scenarios below. For an excellent in-depth review article on Triton and its origin we refer to McKinnon et al. (1995).

\footnotetext{
${ }^{*}$ Corresponding author

Email addresses: erica.nogueira@on.br (E. Nogueira), brasser_astro@yahoo.com (R. Brasser), rodney@on.br (R. Gomes)
} 


\subsection{Capture}

Littleton (1936) hypothesised that Triton and Pluto originated as adjacent prograde satellites of Neptune and that ejection of the latter left the former on a retrograde orbit. Yet McKinnon (1984) demonstrated that this scenario is impossible: the amount of mass and angular momentum in the system is insufficient to make Triton retrograde. McKinnon (1984) argued instead that Triton and Pluto originated from a reservoir of bodies in the outer solar system. Triton was captured by Neptune during a close approach and its orbit was circularised through tidal evolution to its current one. McKinnon (1984) concluded that the tidal heating that followed as Triton circularised should have melted it. However, McKinnon's (1984) capture scenario presented a problem. In order to capture Triton it needs to lose enough energy to be bound from hyperbolic orbit and not be able to subsequently escape through the Hill sphere. This is difficult to do in one orbit using tidal interaction alone. Therefore Benner \& McKinnon (1995) studied temporary capture from heliocentric orbit and the subsequent evolution of a set of these temporary-captured orbits in the circular restricted threebody problem consisting of the Sun, Neptune and a massless Triton. Solar perturbations acting on Triton cause its angular momentum to oscillate with a period half of Neptune's orbital period, with secular perturbations acting on longer time scales. In extreme cases these perturbations align and decrease Triton's pericentre distance, $q$, to within a few Neptune radii. Here the collision with an existing satellite or aerodynamic drag from a putative circumplanetary nebula could have dissipated enough energy to make capture permanent. However, Benner \& McKinnon (1995) favour prompt capture at low $q$ by collision or gas drag because a long-lasting temporary capture would have resulted in large changes in $q$ and thus decreasing the probability of a collision or the effect of gas drag. A prompt capture ensures there are many more close flybys which speed up the reduction of the orbit. In any case, permanent capture was difficult to achieve.

A different scenario for the capture of Triton that did not require complex dynamics was proposed by Goldreich et al. (1989), who favoured the idea of a collision having occurred between Triton and a hypothetical regular satellite of Neptune. Goldreich et al. (1989) argued that a collision with a regular satellite containing a few percent of Triton's mass would have shattered the satellite and left Triton bound to Neptune. However, this scenario could be problematic. The collision probability of Triton with a regular satellite is $10^{-5}$ per pericentre passage. In order to make this scenario work with a reasonable probability, Goldreich et al. (1989) argue there had to be 10000 Triton-mass objects encountering Neptune within $10 R_{N}$, which is too large by several orders of magnitude compared to recent estimates derived from the size-distributions of the Kuiper Belt and Jupiter's Trojans (Morbidelli et al., 2009). However, it may not be necessary that each of these pericentre passages within $10 R_{N}$ has to come from a unique object. Instead, there just have to be of the order of $10^{5}$ such passages of Triton-like objects. The question then becomes whether this many passages is feasible. We shall return to this problem in the next section when we discuss our numerical simulations. Further criticism of this scenario came from McKinnon et al. (1995), who argue that unless the satellite that Triton would collide with is tiny and contains less than $2 \%$ of the mass of Triton, Triton would have shattered too. Since a collision with the larger satellites is much more likely, and assuming that Neptune's putative regular satellites were similar in size and mass to those of Uranus, neither the satellite nor Triton would have survived the collision.

Given the difficulties of permanently capturing Triton in the above scenarios, Agnor \& Hamilton (2006) suggested a new idea. They developed analytical arguments and used numerical simulations to show that Triton could be captured through the dissociation of a binary planetesimal when it passed close to Neptune. These three-body encounters will disrupt the binary if its centre of mass passes close enough to Neptune that the binary's orbital separation is approximately equal to its Hill sphere. Agnor \& Hamilton (2006) tested this scenario with binaries consisting of objects with masses $1 m_{T}$ and $0.1 m_{T}$, where $m_{T}$ is the mass of Triton. Agnor \& Hamilton (2006) showed that capture is plausible for a large variety of initial conditions of the binary, such as the velocity at the time of encounter with Neptune and the separation of the binary. It turned out that the probability to capture Triton decreased rapidly once the encounter velocity exceeded about $10 \%$ of Neptune's orbital velocity, while the lighter body was captured for encounter velocities of up to $70 \%$ of Neptune's orbital velocity. This result is not surprising because the change in velocity at disruption experienced by the heaviest body, $\Delta v_{1}$, is $\Delta v_{1} \propto m_{2}$, while for the lighter body $\Delta v_{2} \propto m_{1}$ (Agnor \& Hamilton, 2006), and thus the lighter body experiences the larger kick and is more easily captured. It then automatically follows that for lighter binaries, while more easily disrupted because of their lower binding energy, it is also more difficult to capture one of its members: the low binding energy translates into a very small $\Delta v$ and one of them is bound to Neptune on a virtually parabolic orbit, where solar perturbations are stronger. Therefore, capture preceded by binary disruption is favoured for heavy binaries or those with a large mass ratio. Agnor \& Hamilton (2006) found that the semimajor axis of Triton upon capture would always exceed $300 R_{N}$, while the lighter body could be captured much closer to Neptune. Unfortunately, Agnor \& Hamilton (2006) do not provide any statistics for their mechanism so that the probability of this event having occurred could not be tested nor compared to other results.

The lack of statistical information about the success rate of capturing Triton through the disruption of a planetesimal binary led Vokrouhlický et al. (2008) to investigate whether or not Triton and the other irregular satellites of the giant planets could be captured via binary dissociation. This project was aimed at providing a comparison with the planet-planet encounter model of Nesvorný et al. (2007). The initial conditions of Vokrouhlický et al. (2008) come from the Nice model (Tsiganis et al., 2005), and they perform a series of simulations of the planetary instability, recording all the close encounters between the planets and planetesimals. They subsequently send a very large 
number of binaries past each planet, with the distribution of the binary's hyperbolic planetocentric orbits taken from the encounter parameters recorded earlier, and their size distribution taken from that of Kuiper Belt objects (e.g. Bernstein et al., 2004). They had difficulty capturing many small satellites from the dissociation of binaries, for reasons that we just explained above. When considering the capture of Triton they conclude that near-equal mass binaries produce the most captures, with a mass ratio of 1:2 to 1:3 preferred. While larger binary primary masses yield more captures, there are fewer of these around so that the overall capture probability decreases. Together with the fact that capturing members from a light binary is more difficult, this could explain why we see no sub-Triton mass irregular satellites orbiting Neptune and Uranus. In conclusion, they find an overall capture probability of less than $2 \%$, with no restrictions on the semi-major axis, eccentricity or inclination. They add that Triton's capture was most likely to have occurred 510 Myr after Neptune's formation when the planetesimal disc was kept dynamically cold by the surrounding gas of the solar nebula; if it was captured during the planetary instability of the Nice model, it is also preferred that it is captured early on before the disc is too dynamically hot and thus the encounter velocity with Neptune is too large.

Thus, it appears that the favoured mechanism for the capture of Triton is through the dissociation of a binary that had a very close encounter with Neptune. After capture, the orbit of Triton needs to shrink to its present size either through tides or other means.

\subsection{Post-capture evolution}

After Triton was captured and gravitationally bound to Neptune, several mechanisms have been invoked to evolve it to its current orbit. These are tidal interaction with Neptune (McCord, 1966; McKinnon, 1984; Goldreich et al., 1989; Correia, 2009), interactions with a circumplanetary gas disc (McKinnon \& Leith, 1995) or a debris disc (Ćuk \& Gladman, 2005).

The first attempt at calculating Triton's orbital history with tidal interaction was performed by McCord (1966), who used the tidal model of MacDonald (1964) and expanded the equations expressing Triton's change in orbital elements up to sixth order in eccentricity. By using reasonable estimates for Triton's Love number $\left(k_{2}\right)$, quality factor $(Q)$ and mass, McCord (1966) concludes that Triton could have reached its current orbit from a highly extended, nearly parabolic orbit within the age of the solar system. The typical time to become circular is some $100 \mathrm{Myr}$ for $Q_{T}=100$, a typical value for rocky bodies.

The results of McCord (1966) were verified by McKinnon (1984) and Goldreich et al. (1989). McKinnon (1984) argues that the tidal dissipation in Triton would have been enough to melt it, increasing the dissipation and thus shortening the time to reach its current orbit. Goldreich et al. (1989), on the other hand, use a simplified tidal model valid only for eccentricity $e \ll 1$, but because of angular momentum conservation they argue that for $e \sim 1$ the tidal evolution only changes the semi-major axis $a$ at constant pericentre, $q$. Assuming a value $k_{2} / Q=10^{-3}$, typical for rocky bodies, they find that Triton could have reached its current orbit from a post-capture orbit with $a=1000 R_{N}$ within $400 \mathrm{Myr}$, but argue that a semimolten Triton would have increased the rate of orbital decay. However, they added that the perturbations from the Sun cause repeated changes in the angular momentum with a period equal to half of Neptune's orbital period. These perturbations in angular momentum cause $q$ to oscillate by as much as $10 R_{N}$ when $a \gtrsim 600 R_{N}$. These oscillations greatly reduce the effect of tides and increase the time to circularise the orbit, but the authors do not specify by how much.

Correia (2009) uses the tidal models of Mignard (1979, 1980) and Hut (1981), which do not require series expansions in the eccentricity, and he includes keeping track of Triton's obliquity and spin rate. By assuming $k_{2} / Q_{T}=10^{-3}$ and an initial spin period of Triton of $24 \mathrm{~h}$, Correia (2009) shows that Triton reaches its current orbit and spin rate in less than 1 Gyr from an orbit with $a \sim 2000 R_{N}$ and $q \sim 7 R_{N}$. In addition, he argues that tides are sufficient to account for Triton's low eccentricity $\left(e \sim 10^{-5}\right)$ and obliquity $\left(\varepsilon_{T}=0^{\circ} .46\right)$. Thus, from the above studies and with reasonable tidal parameters, it appears as if tides raised on Triton by Neptune are capable of reducing it to its current orbit from a post-capture one well within the age of the solar system, provided that Triton's pericentre upon capture is very close to Neptune $\left(q \sim 7 R_{N}\right)$.

A different approach to reducing Triton's orbit from an extended post-capture one was performed by McKinnon \& Leith (1995), who studied the influence of a circum-Neptunian gas disc on the orbit of a post-captured Triton. They mimic a putative nebula around Neptune after a minimum-mass Uranus nebula. They report strong evolution of the eccentricity and semi-major axis but almost no change in the inclination. Their results are insensitive to the radial surface distribution. Thus Triton could have evolved to its current orbit through gas drag and subsequent tidal evolution because, after the gas has disappeared, Triton's eccentricity is about 0.2. Gas drag is able to reduce Triton's orbital angular momentum to its current level in about 1000 years in the absence of solar perturbations. The latter can increase the gas drag time scale to $10^{4}-10^{5}$ years, so that Triton could have outlived a hot, turbulent nebula lasting some 1000 years, but not a cool, long-lived low-mass one $\left(10^{6} \mathrm{yr}\right)$. In addition, McKinnon \& Leith (1995) argue that Triton could have cleared an annulus in the gas that could have halted its orbital decay. Thus, even though gas drag seems a favourable mechanism to circularise Triton from a post-capture orbit, it requires sensitive timing and the role of solar perturbations might reduce its impact. Recently, Ayliffe \& Bate (2009) have performed the most sophisticated simulations of circumplanetary gas discs and they do not encounter circumplanetary discs around protoplanets with masses similar to Uranus and Neptune. This may not be a problem for Neptune because of the existence of Triton. However, Uranus' satellite system exhibits properties that are suggestive of a disc origin. The latter was made popular by Canup \& Ward (2006) who discovered 
that the typical mass ratio of the regular satellites relative to the giant planets should be $\sim 10^{-4}$. The Uranian satellites are all approximately an order of magnitude less massive than that (Jacobson et al., 1992), suggesting a possible different origin than the one advocated by Canup \& Ward (2006). One alternative is that the satellites formed through the viscous spreading of an impact-generated debris disc (Ward \& Canup, 2003). In another alternative Ayliffe \& Bate (2009) suggest that a protoplanet's rotating envelope must cool following the dispersal of the encompassing circumstellar disc. As it does so it may well flatten into a disc suitable for satellite growth, suggesting a relatively late formation of the satellites. In any case, further study is needed to understand the formation of the Uranian satellite system and the idea that Triton was captured by gas drag.

Both the tidal evolution model and the gas drag model have the disadvantage that they are most effective very close to Neptune. However, it is likely that after capture Triton's semi-major axis was very large, $a \gtrsim 100 R_{N}$. Beyond this distance, solar perturbations become important. Specifically, the Sun induces perturbations in Triton that are the same as the Kozai effect experienced by high-inclination asteroids when perturbed by Jupiter (Kozai, 1962). The Kozai mechanism conserves the $z$-component of the angular momentum, $l_{z}=\sqrt{1-e^{2}} \cos I$, where $I$ is the orbital inclination of Triton with respect to the orbit of the perturbing body (the Sun, or Neptune's orbital plane). Hence for prograde orbits inclination and eccentricity oscillate out of phase, while for retrograde orbits these oscillations are in phase. Depending on the initial conditions of the orbit, the Kozai effect can greatly increase the pericentre distance of Triton after capture. In turn, this could greatly lengthen the time it takes for tides or gas drag to circularise the orbit. These problems were pointed out by Ćuk \& Gladman (2005). They argued that the effect of the Kozai oscillations of Triton's pericentre increases the time scale to reach a circular orbit beyond the age of the solar system and therefore tides alone cannot circularise Triton. They reached their conclusion by averaging a simplified version of the tidal equations over one Kozai cycle. In order to find a different and faster mechanism to circularise Triton they investigate the role of an eccentric, retrograde Triton on Neptune's putative regular satellites. Ćuk \& Gladman (2005) argue that an eccentric Triton forces a high eccentricity on Neptune's putative regular satellites, which begin to cross each other and collide within a few thousand years. The collision would shatter both of these regular satellites and create a debris disc that Triton would pass through. By modelling the effect of the disc as a series of impulsive kicks occurring at pericentre, they conclude that the time scale for the evolution of Triton is $\sim 10^{5} \mathrm{yr}$, much shorter than for tides. The natural course of events is for Triton to sweep up all of the mass in the disc through collisions. The added benefit of this fast circularisation is that it could save Nereid from being ejected or colliding with Neptune.

Thus, the debris disc of Ćuk \& Gladman (2005) seems an interesting alternative to the tidal or gas drag models. Unfortunately they do not specify in detail how they modelled the action of the debris disc on Triton so that its results cannot be verified. In addition, their arguments might not hold because of the following. When considering Uranus' satellite system, perturbations from Triton would increase their eccentricities to orbit-crossing values and their inclinations to a few degrees. Using the formulation of Öpik (1976), the collision probability between any of the regular satellites is also $10^{-5}$ per orbit. Since the orbital period of the regular satellites is much shorter than that of Triton just after its capture, these satellites should suffer several collisions before any of them hits Triton. Most of these mutual collisions occur at impact velocities approximately 1 to 2 times the escape velocity of the satellites. Agnor \& Asphaug (2004) show that collisions with such a low mutual velocity tend to be either merging or hit-and-run collisions. For most of these hit-and-run collisions, the ejecta would be approximately $10 \%$ of the mass of the smallest satellite that is involved. Perturbations from the other satellites and Triton will quickly increase the eccentricities of the ejecta and most of these are rapidly swept up. It is likely that eventually a collision with Triton will occur that will shatter both the satellite and Triton. To summarise, it is not immediately clear whether these mutual collisions among the satellites will create the debris disc that Ćuk \& Gladman (2005) suggested, or whether the satellites will remain largely intact and the system is destroyed by a collision with Triton.

In addition to a possible collision, there is another outcome: ejection of Triton. The forcing of the eccentricities and inclinations of the regular satellites by Triton depends on both the eccentricity of Triton and the semi-major axis ratio between Triton and the satellites. For large semi-major axis ratios the eccentricities of the satellites could remain small enough to avoid crossing. Every time Triton passes through the satellite system it receives a root-mean-square energy kick of the order of $\Delta(1 / a) \sim G m_{T} / a_{s}$ (Fernández, 1981; Duncan et al., 1987), where $m_{T}$ is the mass of Triton and $a_{s}$ is the semi-major axis of a satellite. The satellites receive an energy kick of the same magnitude. These kicks cause Triton to random walk in energy. Typically $a_{s} \sim 10 R_{N}$ and so $\Delta(1 / a) \sim 2 \times 10^{-5} R_{N}^{-1}$. For a captured orbit similar to that of Nereid $1 / a \sim 4 \times 10^{-3} R_{N}^{-1}$ and the number of orbits to random walk to ejection is then around 40000 , ignoring a possible rare Lévy flight, and thus the time to eject Triton is some $10^{5}$ years. These are crude estimates but what is important to notice is that the ejection time and the collision time are very similar. Since most collisions leave the majority of the mass in the satellites instead of debris, ejection becomes a feasible outcome. Clearly further study is needed to determine the most likely scenario when considering a putative regular satellite system of Neptune being perturbed by a just-captured Triton.

\subsection{Current approach}

In this paper we investigate the capture and subsequent evolution of Triton in more detail, building on some of the earlier works. First, we study the capture of Triton using the binary capture scenario of Agnor \& Hamilton (2006), but in the framework of the Nice model, as in Vokrouhlický et al. (2009). This 
model has booked a large number of successes, such as explaining the delay that caused the Late Heavy Bombardment of the terrestrial planets (Gomes et al., 2005), the origin of Jupiter's Trojans (Morbidelli et al., 2005), the structure of the Kuiper belt (Levison et al., 2008b), the dichotomy of the asteroid belt (Levison et al., 2009) and the irregular satellites of the giant planets (Nesvorný et al., 2007). Like Vokrouhlický et al. (2009) we re-enact a series of encounters with binary planetesimals. This will give us the distribution of orbits of Triton just after capture. Second, we use the tidal equations of Correia (2009), based on the model of Mignard (1979, 1980), to determine which of the captured orbits are able to circularise within the age of the solar system, and which initial conditions will place Triton approximately $14 R_{N}$ away from Neptune on a circular orbit. We improve upon Correia's (2009) tidal model, as well as those of McCord (1966) and Goldreich et al. (1989), by adding the perturbations of the Kozai mechanism caused by the Sun, as suggested by Ćuk \& Gladman (2005), as well as the perturbations from Neptune's figure. The latter should suppress the Kozai effect once the semi-major axis reaches $a_{c} \sim 73 R_{N}$ (Kinoshita $\&$ Nakai, 1991). The final outcome of the capture and tidal simulations should give us a probability to obtain a circular, retrograde Triton. This probability is then translated into the expected number of Triton-like objects in the proto-planetary disc for consistency. This paper is divided as follows: in section 2 we present our models and employed methods. Section 3 contains the results from the numerical experiments. In section 4 we discuss how Triton's capture affects Neptune's irregular satellites, concentrating on Nereid. Conclusions and summary follow in the last section.

\section{Model and methods}

The model that we have employed consists of three parts. First, we record the encounters of planetesimals with Neptune during a Nice model simulation. Second, the deepest encounters are re-enacted with binaries with varying mass ratio to determine the capture probability and the resulting orbital distribution of captured objects. Third, the orbits of the captured orbits are evolved under the action of tides to determine which ones end up being similar to Triton. Below we explain each stage in detail.

\subsection{Planet migration}

We consider a system consisting of the Sun, the four giant planets and a planetesimal disc. The initial solar system was more compact than today, and the planets are thought to have formed between 5 and 15 AU on quasi-circular, coplanar orbits (Tsiganis et al., 2005). We took the initial conditions of Gomes et al. (2005) which ensured that Jupiter and Saturn crossed their 2:1 orbital resonance some 600 Myr after their formation, which subsequently triggered the planetary instability (Gomes et al., 2005). We assumed that the planetesimal disc was situated just beyond the orbits of the planets, ending at $30 \mathrm{AU}$, with radial mass distribution varying as $r^{-1}$. The planetesimal disc was composed of 10000 equal-mass bodies with

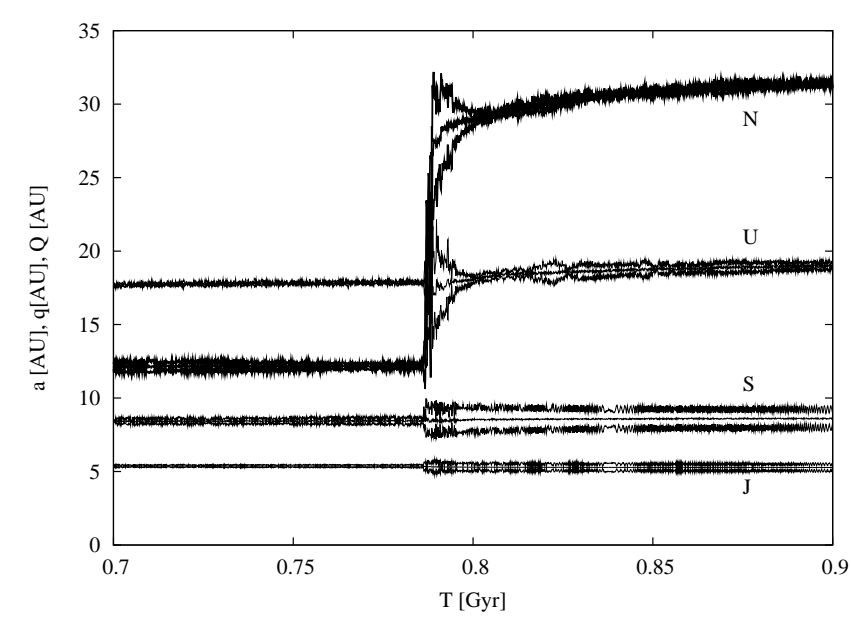

Figure 1: Semi-major axis, perihelion and aphelion distance of the four giant planets around the instability. The letters designate which lines correspond to which planet. Neptune's path is traced with thick lines.

a total mass equal to $35 M_{\oplus}$ (where $M_{\oplus}$ is the Earth's mass). We do not use the initial conditions that arise from the previous phase of migration induced by the gas disc (Morbidelli et al., 2007) because we have a larger database of simulations of the former, and because the interactions between planets and planetesimals are similar in both discs after the late instability. We simulated the dynamical evolution using the numerical integrator MERCURY (Chambers, 1999), where we use a computational 'trick' to decrease the amount of CPU time during the planetary migration simulations (Gomes et al. 2004). We defined an encounter to occur when the distance between a planetesimal and a planet is less than $d=f R_{H}$, where $R_{H}$ is the planetary Hill radius and $f$ is a factor larger than unity. We used $d=1$ AU. Each encounter within this distance was registered in detail, keeping track of the position and velocity of the planetesimal and the planet in the heliocentric reference frame, and the time of closest approach. Once a planetesimal entered the planet's Hill sphere, the system switched to planetocentric coordinates, where it was observed that, as expected, most of the encounters are hyperbolic. However, some of these encounters are elliptical and therefore the planetesimal remains around the planet for a relatively long time during a temporary capture. The total integration time was $4.5 \mathrm{Gyr}$ with a time step equal to $0.4 \mathrm{yr}$.

We performed several Nice model simulations and chose the one where the final orbits of the giant planets are closest to their current ones. Right from the beginning, some planetesimals from the disc encounter the outermost planet and consequently the planets slowly migrate, increasing their orbital separation. This slow leakage from the disc continues until after 788 Myr when Jupiter and Saturn cross the 2:1 resonance. This resonance crossing causes the system to destabilise and one of the ice giants (Neptune) is scattered into the disc, destabilising it, and scattering planetesimals all over the solar system. In our preferred simulation, Saturn and the ice giants undergo many mutual encounters and the ice giants exchange orbits. The 


\begin{tabular}{ccccc} 
Planet & Number & Total enc & Elliptic & Hyperbolic \\
\hline Jupiter & 5866 & 216921 & 8844 & 208077 \\
Saturn & 8249 & 288213 & 4874 & 283339 \\
Uranus & 9891 & 1375907 & 10997 & 1364910 \\
Neptune & 9934 & 1748867 & 65232 & 1683635
\end{tabular}

Table 1: First column: the total number of unique planetesimals that had close encounters with this planet. Second column: the total number of close encounters between planetesimals and this planet. Third column: the total number of elliptical encounters between a planetesimal and this planet. Fourth column: the total number of hyperbolic encounters between a planetesimal and this planet.

number of planetesimals having close encounters with the planets decreased quickly after the instability, and their population decays approximately exponentially. Some $50 \mathrm{Myr}$ after the instability the planets are in their actual configuration. Fig. 1 plots the evolution of the four giant planets around the time of the instability. The lines represent their semi-major axis, perihelion and aphelion distance respectively. The letters designate which set of lines corresponds to which planet: Jupiter stays around 5 AU, Saturn around 9 AU, Uranus goes from 18 AU to $20 \mathrm{AU}$ and Neptune (outlined in thick lines) jumps from $12 \mathrm{AU}$ to $30 \mathrm{AU}$.

The encounter data from this preferred simulation is listed in Table 1. The first column lists the planet with which the encounters occurred. The second column lists the number of unique test particles, out of 10000 , that suffered an encounter with this planet during the lifetime. The third column lists the total number of planetesimals that encountered the planet within 1 AU. The fourth column lists the number of encounters that were elliptical while the last column lists the number of hyperbolic encounters. There are several things that should be pointed out. The first is that Uranus and Neptune undergo many more encounters than Jupiter and Saturn, because the former are scattered into the pristine planetesimal disc while Jupiter and Saturn are left behind. The number of unique particles each planet encounters is similar to the ratios described in Fernández (1997) based on planetesimals that were scattered by Neptune from the Kuiper belt. Specifically, Jupiter typically ends up controlling $58 \%$ of the planetesimals, Saturn approximately $22 \%$ (but it encounters $85 \%$ of them) and Uranus and Neptune approximately $10 \%$ each (so that each will encounter about $90 \%$ of the planetesimals). A second feature is that Neptune encounters substantially more planetesimals than Uranus, partially because some particles that are under its control never encounter Uranus and instead suffer many encounters with Neptune on their way to ejection, and partially because the proximity of Saturn to Uranus decreases the dynamical influence of the latter. Vokrouhlický et al. (2008) also reported a substantially lower number of planetesimal encounters with Uranus than with Neptune. Last, the significantly larger number of elliptical encounters with Neptune vs Uranus is most likely the result of the higher encounter velocities of the planetesimals with Uranus than with Neptune.
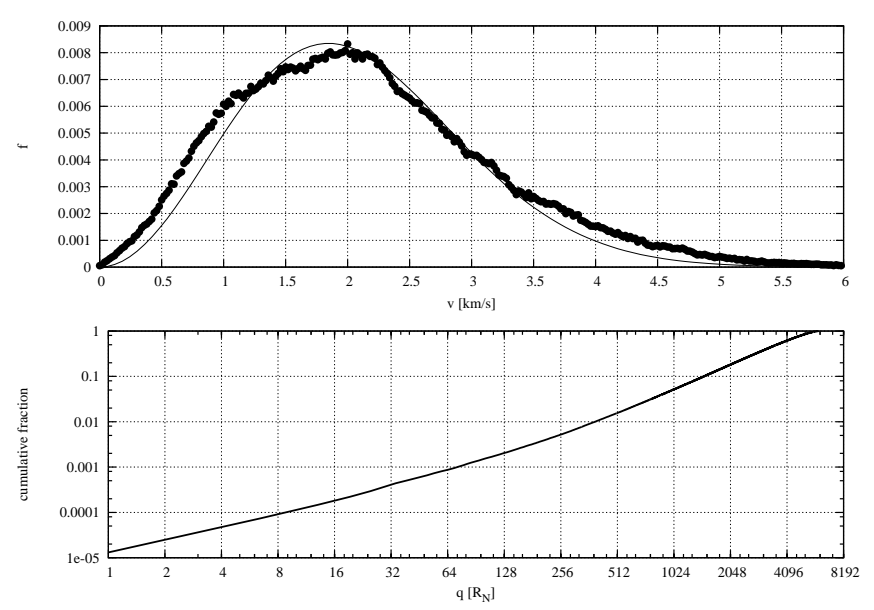

Figure 2: Top panel: velocity distribution of particles that encounter Neptune after the instability. The raw data is shown by the bullets while the solid line shows the best-fit Maxwellian with $v_{\mathrm{m}}=1.31 \mathrm{~km} \mathrm{~s}^{-1}$. Bottom panel: cumulative distribution in pericentre distance with respect to Neptune.

For our purpose we are only interested in planetesimal encounters with Neptune after the instability. For this study, we analyse the distribution of the planetocentric orbits of the planetesimals that had close encounters with Neptune only. Specifically, we are interested in the velocity distribution of the planetesimals as they encounter Neptune and the distribution of their closest approach distance to Neptune. It turns out that the velocity distribution of the encounters is roughly Maxwellian, whose functional form is given by

$$
p(v)=\sqrt{\frac{2}{\pi}} \frac{v^{2}}{v_{\mathrm{m}}^{3}} \exp \left(-\frac{v^{2}}{v_{\mathrm{m}}^{2}}\right),
$$

with $v_{\mathrm{m}}$ the parameter velocity, which was found to be $1.31 \mathrm{~km} \mathrm{~s}^{-1}$. In the top panel of Fig. 2 the bullets depict the velocity distribution of the planetesimals as they encounter Neptune. The best-fit Maxwellian is plotted as a solid line. The bottom panel of Fig. 2 plots the cumulative distribution of the peri-Neptune distance, $q$. For small distances the cumulative distribution is linear in $q$ while beyond $200 R_{N}$ the fit scales as $q^{2}$, suggesting that gravitational focusing is only important for very close encounters. Both plots are in good agreement with those presented in Vokrouhlický et al. (2009).

The cumulative $q$ distribution in the bottom panel of Fig. 2 can be used to constrain the collisional capture scenario of Goldreich et al. (1989), which was mentioned in the introduction. Given that the probability of Triton colliding with a fictitious Neptunian regular satellite is approximately $10^{-5}$ for each periNeptune passage, one needs of the order of $10^{5}$ passages of Triton-like objects within approximately $20 R_{N}$ in order to capture it through a collision event. From the bottom panel of Fig.2 the probability per encounter of a planetesimal coming within $20 R_{N}$ is of the order of $2 \times 10^{-4}$. Combined with the intrinsic collision probability with these fictitious satellites Neptune needs to undergo of the order of $10^{9}$ encounters with Tritonlike objects. Using the data from Table 1 as guidance, on av- 
erage each planetesimal undegoes about 100 encounters with Neptune, so that for each Triton-like object the probability of collisional capture is approximately $10^{-7}$. This low probability makes the collisional capture scenario seem a very unlikely mechanism to account for the existence of Triton.

Now that we have a data base of encounters with Neptune, the next step is to re-enact these encounters using binaries and determine which binary systems are disrupted and also leave Triton bound to Neptune.

\subsection{Binary encounters}

After recording the number of encounters with Neptune from the migration simulation, we re-enacted these using binary systems. The centre of mass of the binary coincides with the trajectory of a planetesimal that had a close encounter with Neptune. From Agnor \& Hamilton (2006) we know that a binary is ionised once the separation between the members becomes equal to the Hill sphere of the binary. This tidal disruption distance is given by

$$
\frac{r_{t d}}{R_{N}}=\left(\frac{a_{B}}{R_{1}}\right)\left[\left(\frac{3 \rho_{N}}{\rho_{1}}\right)\left(\frac{m_{1}}{m_{1}+m_{2}}\right)\right]^{1 / 3} \approx \frac{a_{B}}{R_{1}},
$$

where $R_{N}$ is Neptune's radius, $R_{1}$ is the radius of the primary binary component (usually Triton's radius), $\rho_{N}$ is the mean density of Neptune, $\rho_{1}$ is the mean density of the primary component of the binary (usually Triton's density) and $m_{1}$ and $m_{2}$ are the masses of the primary and secondary of the binary. The approximation sign in equation (2) above is valid when considering densities appropriate for Neptune and Triton. We created four different groups of binaries with different mass ratios, with each binary containing one member with a mass equal to Triton's mass. The other member was either $0.1 m_{T}, 0.3 m_{T}, 1 m_{T}$ or $3 m_{T}$. All binaries had an initially circular orbit and semimajor axis $a_{B}=1.5 R_{N}=37146 \mathrm{~km}$, which is the approximate maximum value for the currently-known trans-Neptunian binary population (Noll et al., 2008). These binaries should disrupt once they come closer to Neptune than $q \sim 27 R_{N}$. The other orbital elements were chosen at random. For each binary group we simulated 1000 different orbits. To be on the safe side, we re-enacted only encounters with $q<100 R_{N}$.

After we created the binary system, we simulated their approach with Neptune using the MERCURY integrator, and registered the changes in semi-major axis, eccentricity and inclination of the binary. We consider that a member is ejected from the system whenever it has $a>3000 R_{N}$. If the pericentre distance is smaller than the planetary radius the body is removed from the simulation.

This part of the model allowed us to create a distribution of orbits that Triton could have had just after capture. The next step is to evolve these orbits using tides raised by Neptune on Triton to determine what fraction of post-capture orbits will yield the current orbit of Triton within the age of the solar system.

\subsection{Tidal evolution}

We decided to use the tidal equations presented in Correia (2009), which are based on the tidal models of Mignard (1979, 1980) and Hut (1981). This model assumes that the time delay of the tidal response of a body, $\Delta t$, is a constant. This model can be made linear in $\Delta t$ and the tidal equations can be written in closed form for all values of eccentricity $e<1$. However, the constant time delay model does not appear to agree with geophysical data (Efroimsky \& Lainey, 2007). The rate of energy dissipation within a body is characterised by the tidal parameter $Q$, which is a measure of how many tidal oscillations are needed to damp the energy by order of itself. The constant time delay model of Mignard $(1979,1980)$ assumes that $Q$ is inversely proportional to the frequency with which the body is distorted, $\chi$. For eccentric orbits $\chi$ is the angular frequency of this body at pericentre. Laboratory experiments indicate that $Q \propto \chi^{\alpha}$, where $\alpha \sim 1$ for the range of periods that we are interested in (Karato, 2008). Nevertheless, we are mostly dealing with orbits with $e \sim 1$ where series expansions are invalid, and thus we decided to adopt this model because it best suits our needs. We decided to use a simplified model in which we integrate the equations governing the changes in semi-major axis, eccentricity, spin rate and obliquity of Triton, while ignoring any changes in the rotation rate of Neptune and its obliquity. This is justified because Correia (2009) found that the change in both of these quantities is negligible. We found that tides raised by Triton on Neptune are a couple of orders of magnitude weaker than tides raised by Neptune on Triton, but we included them for the sake of completeness. The tidal equations are given by (Correia, 2009)

$$
\begin{aligned}
\dot{a}_{T} & =\frac{2 K_{T}}{m_{T} a_{T}}\left(\frac{f_{2}\left(e_{T}\right) \cos \varepsilon_{T} \omega_{T}}{n_{T}}-f_{3}\left(e_{T}\right)\right) \\
& +\frac{2 K_{N}}{m_{T} a_{T}}\left(\frac{f_{2}\left(e_{T}\right) \cos i_{T} \omega_{N}}{n_{T}}-f_{3}\left(e_{T}\right)\right) \\
\dot{e}_{T} & =\frac{9 K_{T} e_{T}}{m_{T} a_{T}^{2}}\left(\frac{11 f_{4}\left(e_{T}\right) \cos \varepsilon_{T} \omega_{T}}{18 n_{T}}-f_{5}\left(e_{T}\right)\right) \\
& +\frac{9 K_{N} e_{T}}{m_{T} a_{T}^{2}}\left(\frac{11 f_{4}\left(e_{T}\right) \cos i_{T} \omega_{N}}{18 n_{T}}-f_{5}\left(e_{T}\right)\right), \\
\dot{\omega}_{T} & =-\frac{K_{T} n_{T}}{C_{T}}\left(f_{1}\left(e_{)} \frac{1+\cos ^{2} \varepsilon_{T}}{2} \frac{\omega_{T}}{n_{T}}-f_{2}\left(e_{T}\right) \cos \varepsilon_{T}\right),\right. \\
\dot{\varepsilon}_{T} & =\frac{K_{T} n_{T}}{C_{T} \omega_{T}} \sin \varepsilon_{T}\left(\frac{f_{1}\left(e_{T}\right) \cos \varepsilon_{T} \omega_{T}}{2 n_{T}}-f_{2}\left(e_{T}\right)\right),
\end{aligned}
$$

where $\varepsilon_{T}$ is Triton's obliquity with respect to its own orbit, $i_{T}$ Triton's inclination with respect to Neptune's equator, $\omega_{T}$ is Triton's spin rate, $\omega_{N}$ is Neptune's spin rate, $n_{T}$ is Triton's mean motion, $C_{T}$ is Triton's moment of inertia along its spin axis, and

$$
\begin{aligned}
K_{T} & =\frac{3 k_{2 T} G m_{N}^{2} R_{T}^{5} \Delta t_{T}}{a_{T}^{6}}, \\
K_{N} & =\frac{3 k_{2 N} G m_{T}^{2} R_{N}^{5} \Delta t_{N}}{a_{T}^{6}},
\end{aligned}
$$




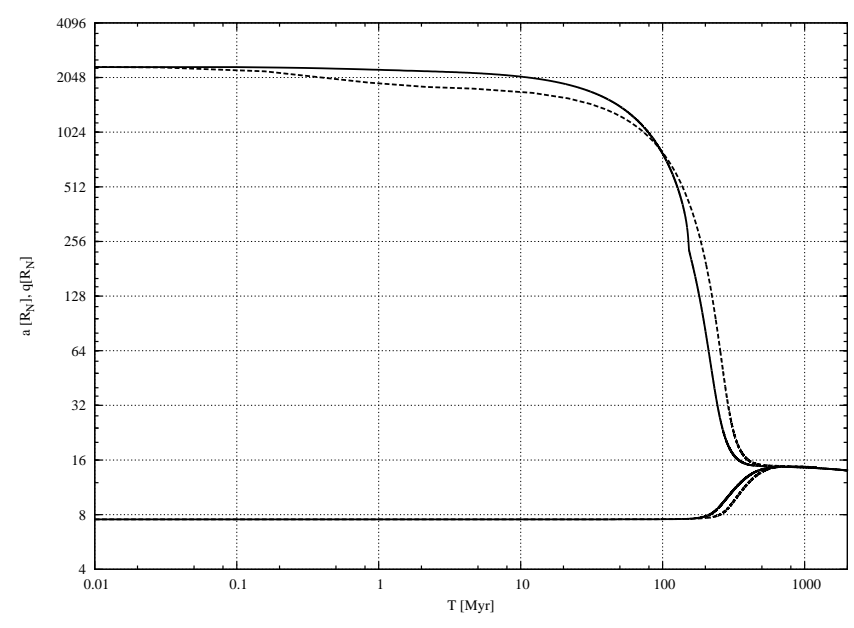

Figure 3: Tidal evolution of semi-major axis and pericentre distance of a Tritonsized object captured by Neptune. Initial conditions are from Correia (2009). Solid lines depict the evolution from Correia (2009) while the dashed lines show our simplified implementation. Solid lines data set courtesy of Alexandre Correia.

$$
\begin{aligned}
f_{1}(e) & =\left(1+3 e^{2}+3 e^{4} / 8\right)\left(1-e^{2}\right)^{-9 / 2}, \\
f_{2}(e) & =\left(1+15 e^{2} / 2+45 e^{4} / 8+5 e^{6} / 16\right)\left(1-e^{2}\right)^{-6}, \\
f_{3}(e) & =\left(1+31 e^{2} / 2+255 e^{4} / 8+185 e^{6} / 16+25 e^{8} / 64\right) \\
& \times\left(1-e^{2}\right)^{-15 / 2} \\
f_{4}(e) & =\left(1+3 e^{2} / 2+e^{4} / 8\right)\left(1-e^{2}\right)^{-5}, \\
f_{5}(e) & =\left(1+15 e^{2} / 4+15 e^{4} / 8+5 e^{6} / 64\right)\left(1-e^{2}\right)^{-13 / 2} .
\end{aligned}
$$

Here $k_{2 T}$ is Triton's Love number $k_{2}$ and $\Delta t_{T}$ is Triton's tidal response time, $R_{T}$ is Triton's radius and $m_{T}$ is its mass. The quantities carrying subscripts $\mathrm{N}$ are for Neptune. Table 2 lists the values of the various quantities used above. Most are taken from Correia (2009), and references therein. The values of $\omega_{T}$ and $\varepsilon_{T}$ are starting values, which evolve to their current ones. An example of the tidal evolution in this simplified model is depicted in Fig. 3. and shows the evolution of semi-major axis and pericentre distance of a Triton-sized object captured by Neptune. The initial conditions are taken from Correia (2009) and are $a_{0}=2354 R_{N}, q_{0}=7 R_{N}, i_{0}=157^{\circ}$ and $\omega_{r 0}=1 / 24 \mathrm{hr}^{-1}$. The solid lines show the evolution depicted in Correia (2009) while the dashed lines show the evolution according to our simplified model. The slight differences are caused by Correia (2009) taking Cassini states and properly taking Triton's obliquity evolution into account.

We considered two values of $\Delta t_{T}$. The first, $\Delta t_{T}=\left(Q_{T} \omega_{T}\right)^{-1}=$ $808 \mathrm{~s}$, where we used the current value of $\omega_{T}$ and corresponds to imposing $Q_{T}=100$, a commonly adopted value for rocky bodies and close to the inferred value for Mars of $Q_{M}=92$ (Yoder et al., 2003). However, Triton was most likely semimolten during its tidal circularisation (McKinnon, 1984), which

\begin{tabular}{|c|c|}
\hline Quantity & Value \\
\hline$m_{T}$ & $1.0837 \times 10^{-8} \mathrm{M}_{\odot}$ \\
\hline$m_{N}$ & $5.156 \times 10^{-5} \mathrm{M}_{\odot}$ \\
\hline$k_{2 T}$ & 0.1 \\
\hline$k_{2 N}$ & 0.407 \\
\hline$R_{T}$ & $9.044 \times 10^{-6} \mathrm{AU}(1353 \mathrm{~km})$ \\
\hline$R_{N}$ & $1.6554 \times 10^{-4} \mathrm{AU}(24764 \mathrm{~km})$ \\
\hline$C_{T}$ & $0.35 m_{T} R_{T}^{2}$ \\
\hline$\omega_{T}$ & $6884.65 \mathrm{rad} \mathrm{yr}^{-1}\left(P_{T}=8 \mathrm{~h}\right)$ \\
\hline$\omega_{N}$ & $3418.82 \mathrm{rad} \mathrm{yr}^{-1}\left(P_{N}=16.11 \mathrm{~h}\right)$ \\
\hline$\varepsilon_{T}$ & $170^{\circ}$ \\
\hline$\varepsilon_{N}$ & $28.56^{\circ}$ \\
\hline$\Delta t_{N}$ & $1.02 \mathrm{~s}\left(Q_{N}=9000\right)$ \\
\hline$\Delta t_{T}$ & $808 \mathrm{~s}$ or $8080 \mathrm{~s}$ \\
\hline$a_{\odot}$ & $30.1 \mathrm{AU}$ \\
\hline$e_{\odot}$ & 0.008 \\
\hline$J_{2}$ & $3.343 \times 10^{-3}$ \\
\hline
\end{tabular}
decreases the value of $Q$ and thus increases that of $\Delta t_{T}$. Using Io as an example, it has $k_{2} / Q_{I} \approx 0.015$ and thus $Q_{I} \sim 3$ for $k_{2}=$ 0.05 (Lainey et al., 2009) but when using the fluid Love number, appropriate for semi-molten bodies, $k_{2}=1.292$ (Anderson et al., 2001) and we have $Q_{I} \sim 86$. For solid bodies, we can turn
Table 2: Values of various quantities that enter the tidal equations, as well as those of the Kozai mechanism and figure of Neptune.

to the Moon and Mars. For the Moon, the inferred value of $k_{2}$ from laser ranging is $k_{2}=0.02664$ and $Q \sim 30$ (Williams et al., 2005). This low value of $Q$ is caused by tidal dissipation within the Moon's core, and results in $k_{2} / Q \sim 0.0011$. For Mars, its value of $k_{2}$ ranges from 0.11 to 0.16 (Marty et al., 2009) while its value of $Q$ ranges between 80 (Lainey et al., 2007) and 92 (Yoder et al., 2003), suggesting that for Mars $k_{2} / Q \sim 0.0015$. Thus, it seems that for rocky bodies $k_{2} / Q \sim 0.0013$ while for semi-molten bodies $k_{2} / Q \sim 0.015$. We decided to adopt an intermediate value of $k_{2}=0.1$ for Triton (McKinnon et al., 1995) and change the value of $Q_{T}$ by a factor ten between the solid and semi-molten state. For the solid state we adopt $k_{2} / Q_{T}=10^{-3}$, implying $Q_{T}=100$, and for the semi-molten state $k_{2} / Q_{T}=10^{-2}$, implying $Q_{T}=10$. This corresponds to a time delay $\Delta t=808 \mathrm{~s}$ in its current orbit for the solid state, and $8080 \mathrm{~s}$ when semi-molten.

As stated in the introduction, the tides are not acting alone. For large orbits, the Kozai mechanism (Kozai, 1962) is at work, caused by perturbations from the Sun, and we need to take its influence into account (Ćuk \& Gladman, 2005). The Kozai mechanism has two constants of motion (Kozai, 1962; Kinoshita \& Nakai, 2007)

$$
\begin{aligned}
H_{K} & =\gamma\left[\left(2+3 e^{2}\right)\left(3 \cos ^{2} I-1\right)+15 e^{2} \sin ^{2} I \cos 2 \omega\right], \\
h_{z} & =\left(1-e^{2}\right)^{1 / 2} \cos I,
\end{aligned}
$$

where we omit the subscript $\mathrm{T}$ since we are only dealing with Triton. Here $H_{K}$ is the averaged Kozai Hamiltonian (Kozai, 1962), $h_{z}$ is the $z$-component of the orbital angular momentum, $I$ is Triton's inclination with respect to Neptune's orbital plane, $\omega$ is Triton's argument of pericentre and

$$
\gamma=\frac{n_{\odot}^{2} a_{T}^{2}}{16\left(1-e_{\odot}^{2}\right)^{3 / 2}} .
$$


The Kozai mechanism induces coupled oscillations in eccentricity and inclination, and either a circulation or libration of $\omega$, depending on the value of $H_{K}$ and $h_{z}$. The equations of motion of these three variables, and $\Omega$, the longitude of Triton's ascending node on Neptune's orbital plane (see below) are (Kinoshita \& Nakai, 2007)

$$
\begin{aligned}
\dot{e} & =\frac{30 \gamma}{n a^{2}} e\left(1-e^{2}\right)^{1 / 2} \sin ^{2} I \sin 2 \omega, \\
\dot{I} & =-\frac{15 \gamma}{n a^{2}\left(1-e^{2}\right)^{1 / 2}} e^{2} \sin 2 I \sin 2 \omega, \\
\dot{\omega} & =\frac{6 \gamma}{n a^{2}\left(1-e^{2}\right)^{1 / 2}}\left[-1+e^{2}+5 \cos ^{2} I\right. \\
& \left.+5\left(1-e^{2}-\cos ^{2} I\right) \cos 2 \omega\right], \\
\dot{\Omega} & =-\frac{6 \gamma}{n a^{2}\left(1-e^{2}\right)^{1 / 2}} \cos I\left(3 e^{2}+2-5 e^{2} \cos 2 \omega\right) .
\end{aligned}
$$

In addition to the Kozai mechanism, the figure of Neptune perturbs Triton's orbit once it is close enough to Neptune. While the perturbations of Neptune's figure do not alter the eccentricity and inclination, it does force a precession of the argument of pericentre. Once Triton is close enough to Neptune, this precession will overtake the precession induced by the Kozai effect and the coupled oscillations in inclination and eccentricity will stop. Thus, in order to model the disappearance of the Kozai mechanism, we need to add the perturbations of Neptune's figure to the tidal model as well. The averaged Hamiltonian is (e.g. Kinoshita \& Nakai, 1991)

$$
H_{J 2}=\frac{1}{4} \frac{G m_{N}}{a} J_{2}\left(\frac{R_{N}}{a}\right)^{2} \frac{\left(3 \cos ^{2} i-1\right)}{\left(1-e^{2}\right)^{3 / 2}},
$$

where $i$ is Triton's inclination with respect to Neptune's equator and $J_{2}$ is Neptune's quadrupole moment. In order to add this to the tidal equations of motion and those of the Kozai mechanism, we need to transform Triton's inclination with respect to Neptune's equator to its inclination with respect to Neptune's orbital plane. This is done via $\cos i=\cos \varepsilon_{N} \cos I-\sin \varepsilon_{N} \sin I \cos \Omega$, where $\varepsilon_{N}$ is Neptune's obliquity. Unfortunately this adds another variable, $\Omega$, to be integrated, and therefore the Kozai part of the regression of this angle needs to be taken into account as well (hence its inclusion above). We have

$$
\begin{aligned}
\dot{I} & =-\frac{3}{2} \frac{G m_{N}}{a} \frac{J_{2}}{n a^{2}\left(1-e^{2}\right)^{2}}\left(\frac{R_{N}}{a}\right)^{2} \cos i \sin \varepsilon_{N} \sin \Omega \\
\dot{\omega} & =\frac{3}{4} \frac{G m_{N}}{a} \frac{J_{2}}{n a^{2}\left(1-e^{2}\right)^{2}}\left(\frac{R_{N}}{a}\right)^{2} \\
& \times\left[\cot I \sin 2 i \frac{d i}{d I}+3 \cos ^{2} i-1\right] \\
\dot{\Omega} & =-\frac{3}{4} \frac{G m_{N}}{a} J_{2}\left(\frac{R_{N}}{a}\right)^{2} \frac{\csc I \sin 2 i}{n a^{2}\left(1-e^{2}\right)^{2}} \frac{d i}{d I}
\end{aligned}
$$

We integrated the system of equations consisting of (3), (7) and (9) using a Bulirsch-Stoer integrator with variable time step (Bulirsch \& Stoer, 1966). Once Triton was closer than $20 R_{N}$ to Neptune, we stopped integrating the Kozai and $J_{2}$ effects because the former is suppressed by the $J_{2}$ precession and the latter only causes circulation of $\Omega$ and $\omega$, which do not contribute to the tidal evolution. Switching off the integration of these quantities significantly sped up the final part of the tidal evolution.

When considering pure tidal motion, the orbital angular momentum of Triton is approximately conserved. Thus, for initially very eccentric orbits, the final, circular, orbit is located at $a \approx 2 q_{0}$, where $q_{0}$ is the original pericentre distance. Triton's current orbit suggests it was captured with $q_{0} \sim 7 R_{N}$. However, the Kozai mechanism induces oscillations in the eccentricity and thus in $q$. From equations (3) we have $\dot{a} \propto q^{-15 / 2}+$ $O\left(q^{-13 / 2}\right)$, so that a typical time scale on which the tides act, $T_{a}=a / \dot{a} \propto q^{15 / 2}+O\left(q^{13 / 2}\right)$ is a very steep function of $q$ and suggests the annulus in which the tides can circularise Triton within the age of the solar system is very narrow. Indeed, increasing $T_{a}$ by an order of magnitude requires a relative increase in $q$ of only $35 \%$. Experimentation showed that the time to become circular reaches the age of the solar system for $Q_{T}=10$ when $q_{0}=20 R_{N}$, and thus orbits for which $q$ never dips below $20 R_{N}$ can be ignored. Given the rapid increase in $T_{a}$ with $q$, in the simplest and crudest sense one can envision the $q$ dependence as a step function, where the tides are switched off if $q$ is larger than some threshold value, and the tides are active when $q$ is smaller. This behaviour suggests that the final orbit of Triton would have $a \approx 2 q_{\min }$, where $q_{\min }$ is the minimum value of $q$ that is obtained during the Kozai cycle. Indeed this appears to be a better approximation than the previous one $a \approx 2 q_{0}$.

Now that we have all the ingredients in place, we report the results of our experiments below. In what follows, we removed any objects that achieved a final semi-major axis $a<5 R_{N}$ since it would then collide with Proteus (located at $4.8 R_{N}$ ), or which encountered $q<1 R_{N}$ during their Kozai cycle, or whose $q$ never reached below $25 R_{N}$ within $1 \mathrm{Myr}$ (by comparison: the Kozai cycle time is typically $0.01 \mathrm{Myr}$ ). While we did integrate cases with $Q_{T}=100$, we do not present the results here since it was most likely that Triton was semi-molten (McKinnon, 1984). In what follows, the time scale for the tidal evolution of Triton should be considered as indicative rather than absolute. This is caused by the lack of knowledge of the tidal parameters and the model's simplification of the real tidal evolution.

\section{Results}

In this section the results from our numerical simulations are presented. First we present the distribution of captured orbits. This is followed by a case study of the tidal evolution, after which we turn to the tidal evolution of all the captured orbits.

\subsection{Captured orbits}

Fig. 4 displays the pericentre distance $(q)$ vs. semi-major axis $(a)$ of objects with Triton's mass that were successfully 


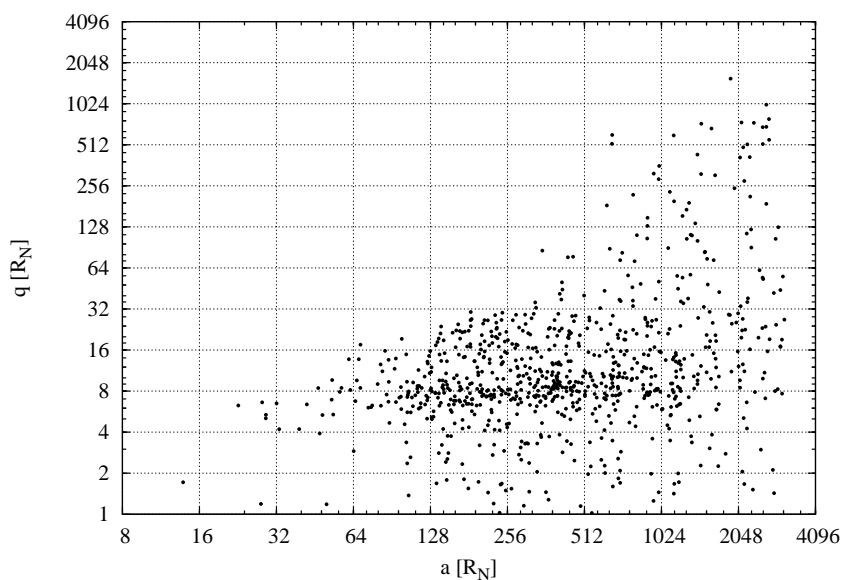

Figure 4: Pericentre distance vs. semi-major axis of captured satellites with Triton's mass after the disruption of a binary. These are orbits only upon capture without subsequent tidal evolution.

captured following the disruption of a binary, as outlined in Section 2.2. Since the binaries were enacted from encounters with Neptune that had $q<100 R_{N}$, it is no surprise that most captured objects have a rather small value of $q$. The truncation of the semi-major axis at approximately $3000 R_{N}$ is caused by our condition that objects with $a>3000 R_{N}$ are considered unbound since they will be outside Neptune's Hill sphere at apocentre. Fig. 5] shows the cumulative distributions of semi-major axis (top-left), pericentre (top-right), inclination (bottom-left) and eccentricity (bottom-right) for Triton-mass satellites upon capture. The steep increase in the distribution of $q$ between $8 R_{N}$ and $32 R_{N}$ is probably caused by our choice of binaries. Similarly, the sharp rise in the eccentricity distribution when $e \gtrsim 0.9$ is might also be an artefact of our initial conditions. The inclination distribution shows there is a clear preference for retrograde orbits upon capture, probably because of the increased stability of retrograde orbits with respect to the size of Neptune's Hill sphere (Hamilton \& Krivov, 1997). In summary, most orbits are captured with $a \in(100,2000) R_{N}$ and $q \in(4,32) R_{N}$ with a clear retrograde preference. In contrast, Agnor \& Hamilton (2006) find that the median semi-major axis of Triton after capture is $a \gtrsim 1000 R_{N}$, while we find a value of $a \sim 500 R_{N}$, suggesting that the encounter parameters when Neptune migrates are different from the static case. These orbits now need to be evolved using the tidal model presented above. We define a successful case when Triton achieves $e \sim 10^{-5}$ in less than $4 \mathrm{Gyr}$. In principle one could define a successful case as any capture for which $e<1$ and $q>1 R_{N}$ but then the current orbit is not reproduced and thus we decided to use the former criterion.

\subsection{Tidal evolution: Case study}

In order to determine whether or not we can obtain Triton's current orbit within the age of the solar system from the captured orbits presented in the previous subsection, we need to run them through the tidal equations. In this subsection we show a representative case of the evolution of Triton upon capture. In
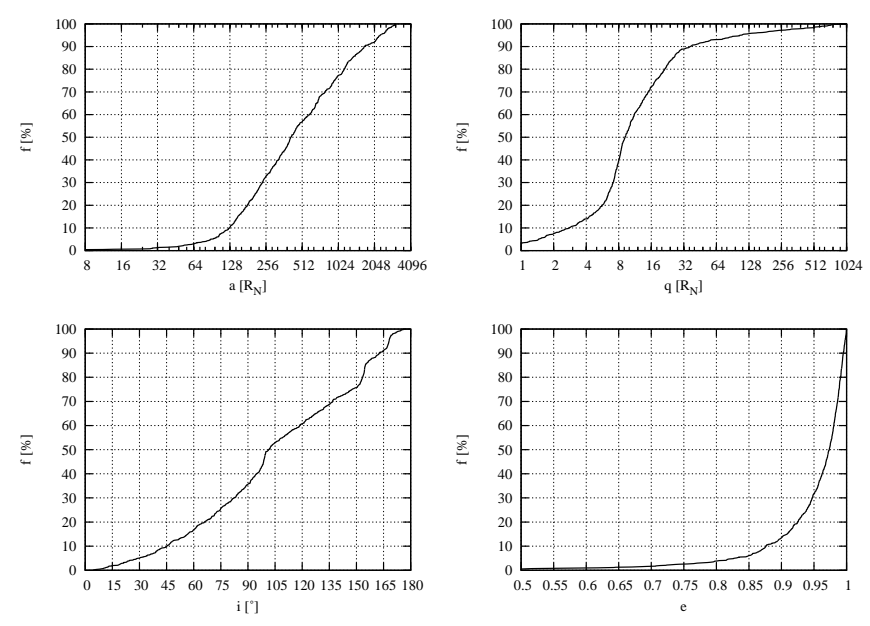

Figure 5: Cumulative distributions of semi-major axis (top-left), pericentre (top-right), inclination (bottom-left) and eccentricity (bottom-right) for Tritonmass satellites upon capture.

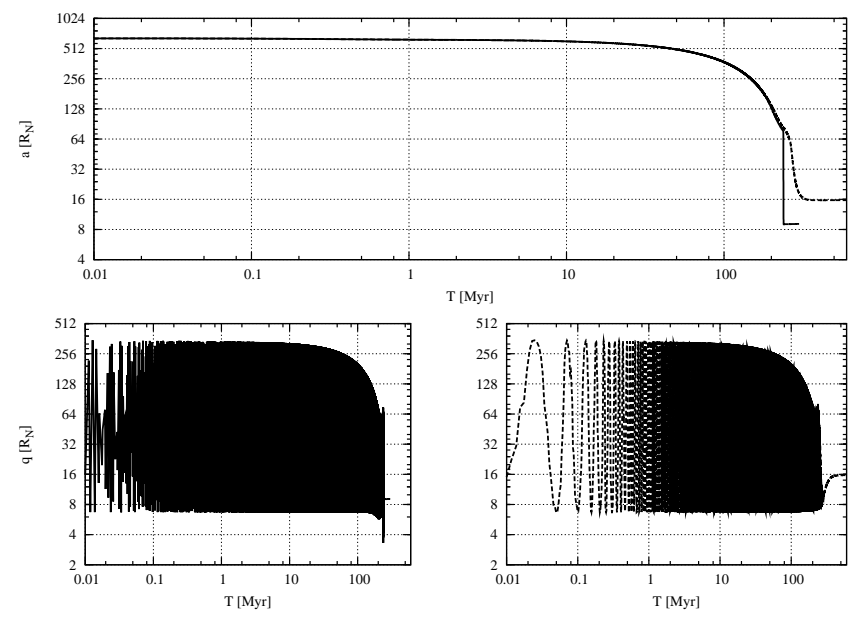

Figure 6: Example of tidal evolution of one object using two different models. The top panel shows the evolution in semi-major axis. The solid line uses Neptune's current obliquity. The dashed line has Neptune's obliquity set to zero. The bottom panels show the evolution of the pericentre. Left panel has the current obliquity of Neptune while the right panel has Neptune's obliquity set to zero.

the next subsection we present the results of the final orbits of all captured objects. We have decided to model the tidal evolution using two different methods, in order to compare them. For the first method, we integrate the tidal equations from section 2.2 using as the initial conditions the captured orbits presented above. We included Kozai and Neptune's figure and set Neptune's obliquity equal to its current value. With this method one can obtain Triton's final inclination with respect to Neptune's current equator. For the second case, we use the same equations as above but set Neptune's obliquity equal to zero. This was done to determine how the Kozai perturbations from the Sun determine the final outcome.

Fig. 6 presents the results of the tidal evolution of one object using $Q_{T}=10$ i.e. the semi-molten case. The starting conditions are $a=648.2 R_{N}, q=8.38 R_{N}, I=52.1^{\circ}, \omega=207.1^{\circ}$. 
The top panel shows the evolution of the semi-major axis vs. time. The solid line depicts the case with Neptune's current obliquity $\varepsilon_{N}=28.56^{\circ}$ (case 1 ). The dashed line depicts the case where the obliquity of Neptune is zero (case 2). In both cases the pericentre distance oscillates with large amplitude and short period compared to the tidal evolution. The latter occurs quickest when $q$ is at a minimum, and when this happens the semi-major axis shrinks. With each decrease in semi-major axis at minimum $q$, the value of $h_{z}$ decreases and the excursions in $q$ (and $e$ ) are less pronounced. Eventually the semi-major axis shrinks to the point where the perturbations from Neptune's figure compete with the Kozai cycles induced by the Sun. This occurs at approximately $70 R_{N}$. Once the semi-major axis shrinks below this value the Kozai cycles cease and the rest of the evolution proceeds at constant angular momentum. However, this value of the angular momentum is not equal to the value upon capture. The final semi-major axis is approximately $a_{\mathrm{f}} \sim 2 q_{\mathrm{min}}$.

Fig. 7 presents a zoom for case 1 around the time when the Kozai cycles stop. The dotted line plots the semi-major axis vs time while the solid line represents the pericentre distance, $q$. The oscillations in $q$ decrease in amplitude and the minima are consistently closer to Neptune. The decrease in the minimum distance of $q$ is caused by the perturbations from Neptune's figure not being aligned with those of the Sun. Far from Neptune the orbital angular momentum vector of Triton precesses perpendicular to Neptune's orbital plane. As the semi-major axis of Triton shrinks, the perturbations from Neptune's figure become stronger compared to the Kozai cycles induced by the Sun. Neptune's equator is not aligned with its orbit and the perturbations from Neptune's figure force Triton's orbital angular momentum to precess perpendicular to Neptune's equator rather than its orbit. The transition happens around $70 R_{N}$. As the angular momentum gradually starts to precess about Neptune's rotational pole, the value of $h_{z}$, which is a constant for the Kozai motion, has to decrease, causing an increase in the maximum eccentricity and thus a decrease in the minimum value of $q$. Eventually the minimum $q$ has dropped close to $4 R_{N}$ and the tidal evolution is then so rapid that the semi-major axis shrinks considerably before Kozai cycles lift the pericentre again. However, by this time, the amplitude of the Kozai cycles have decreased considerably so that future cycles are quickly damped and the semi-major axis continues to decrease. By now the tidal evolution more or less conserves the current total angular momentum since the Kozai cycles have stopped, and the orbit circularises at $a \sim 2 q_{\text {min }}$.

The outcome for the two simulations are different and are presented in Table 3. The first column represents the elements $a, q$ and time to become circular $\left(T_{c}\right)$. The second column lists the initial values. The third column marks the first case (current obliquity of Neptune). The fourth column marks the second case (no obliquity of Neptune). As one can see, the first case yields an orbit much closer to Neptune than the second case. The time to become circular varies by a factor of two. The difference between the final semi-major axis and values of $T_{c}$ are the result of the difference in Neptune's obliquity. For the first

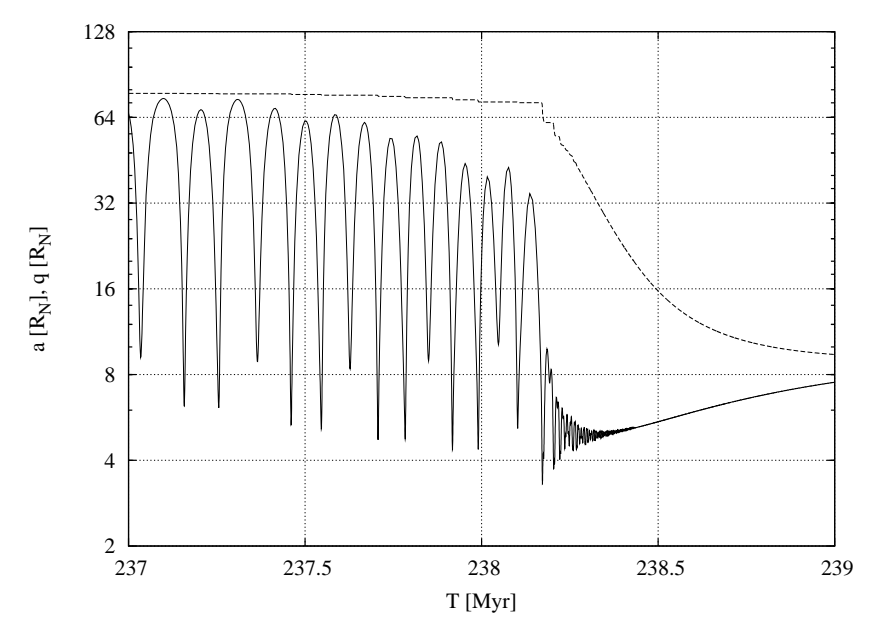

Figure 7: Zoom of the tidal evolution of Fig. 6around the time the Kozai mechanism stops. The dashed line shows the semi-major axis vs time while the solid line shows the pericentre distance. The minimum $q$ decreases, due to a misalignment between Neptune's rotational pole and orbital pole, until eventually it reaches below $4 R_{N}$. The tidal evolution is then so rapid that the semi-major axis shrinks sufficiently for the Kozai cycles to stop. The remaining evolution proceeds at constant angular momentum.

\begin{tabular}{cccc} 
Element & Initial & 1 & 2 \\
\hline & & & \\
$a$ & 648.2 & 9.03 & 15.68 \\
$q$ & 8.38 & 9.03 & 15.68 \\
$T_{c}$ & 0 & 242.3 & 631.8
\end{tabular}

Table 3: The initial and final semi-major axis $(a)$, pericentre $(q)$ and time to circularise $\left(T_{c}\right)$ for the sample orbit with two different tidal models. The column (1) depicts the fist case where Neptune has its current obliquity. The column (2) corresponds to the case where Neptune's obliquity is zero.

case the orbital evolution proceeds very quickly once $q \sim 4 R_{N}$, which does not occur in the second case, where $q$ never drops below $\sim 7 R_{N}$.

Now that we have given an overview of the tidal evolution, we turn to what the final solutions are when the ensemble of captured orbits are run through the tidal model.

\subsection{Tidal evolution: Final orbits}

In this subsection we shall present the results of the possible final orbits of Triton after tidal evolution of the captured orbits. We shall focus on those cases that reach $e=10^{-5}$ within the age of the solar system. Other cases are discarded since they are incompatible with Triton's current orbit.

Fig. 8 shows the cumulative distribution in semi-major axis of those objects that reach Triton's current eccentricity within the age of the solar system for the semi-molten case $\left(Q_{T}=10\right)$. The solid line is for case 1 , the dashed line represents case 2 (indicated on the plot). There are two important features to note here. First, case 1 yields many orbits with a small final semimajor axis because of the feature displayed in Fig.7 7 above: the minimum value of $q$ decreases as $a$ decreases and the orbits circularise at approximately $2 q_{\min }$. The second feature is that 


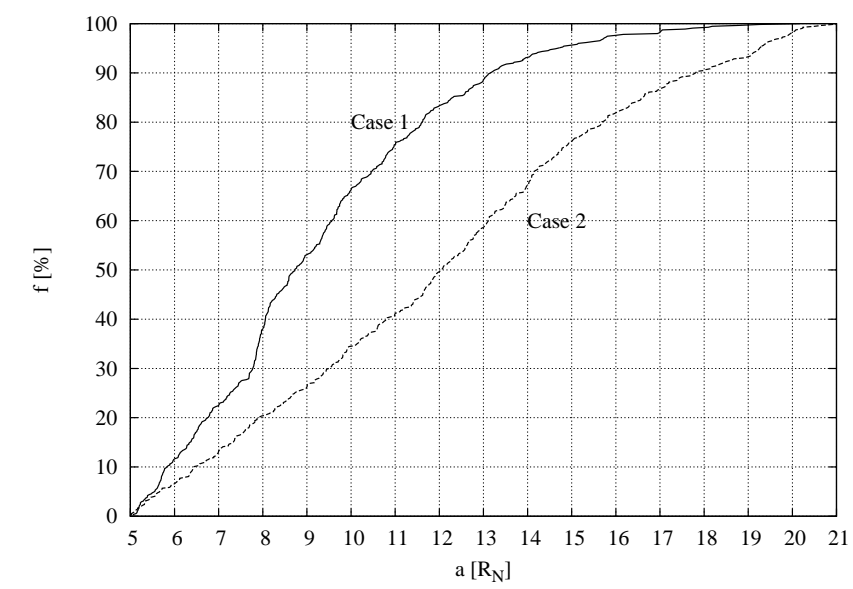

Figure 8: Cumulative distribution of final semi-major axis for objects which reach $e=10^{-5}$ within the age of the solar system. The solid line is for case 1 , the dashed line represents case 2 .
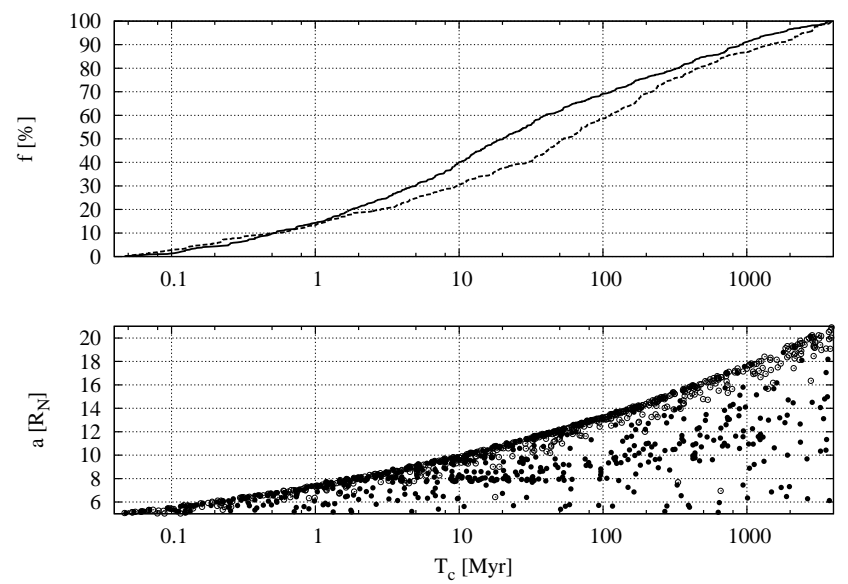

Figure 9: Top panel: Cumulative distribution of time to become circular. The solid line is for case 1 , the dashed line represents case 2 . Bottom panel: time to become circular vs. final semi-major axis. Bullets present case 1, open circles are case 2. The border between the filled and open spaces of the plot scales approximately as $a_{f} \propto T_{c}^{2 / 15}$.

Triton's current orbit at $14.3 R_{N}$ is always in the upper quartile of the distribution; in the worst case it is in the upper $5 \%$ of the distribution. This result would suggest that if tides were the dominant mechanism behind circularising Triton's orbit after capture, one would think that Triton should be closer to Neptune than it is today.

Fig.9 depicts the cumulative distribution of the time it takes for Triton to become circular (top panel) and the final semimajor axis vs. circularisation time in the bottom panel. Once again in the top panel the solid line presents case 1, the dashed line is case 2 . As one can see, case 1 has a much shorter circularisation time than case 2 . This is no surprise because the circularisation time depends heavily on $q$ as $T_{c} \propto q^{15 / 2}$ and since case 1 experiences the lowest values in $q$ it should circularise the quickest. From the plot one can infer that the median time to become circular ranges from $30 \mathrm{Myr}$ to $70 \mathrm{Myr}$ depending on the tidal model that is used. However, care has to be taken when using this number because it does not specify the final value of semi-major axis that is obtained. For that we turn to the bottom panel, which depicts the final semi-major axis vs. the circularisation time. The bullets present case 1, the open circles are case 2. The line between the filled and open parts of the panel scales as $a_{f} \propto T_{c}^{2 / 15}$. For Triton at $14 R_{N}$, the time to become circular by tides ranges from approximately $200 \mathrm{Myr}$ for most orbits, up to $4 \mathrm{Gyr}$ for a few exceptional cases, depending on the configuration of the original orbit. Thus, Triton would have reached its current orbit approximately $200 \mathrm{Myr}$ after its capture. This time scale is consistent with earlier results of McCord (1966), McKinnon (1984), Goldreich et al. (1989), McKinnon et al (1995) and Correia (2009), who all give a typical time scale of 100$500 \mathrm{Myr}$ for Triton to become circular, even though not all of the above works use the same tidal model as ours, nor do they take the Kozai mechanism into account. However, our tidal circularisation time scale is different from that predicted by Ćuk \& Gladman (2005), even if it agrees with other estimates in the literature. We can think of two reasons for this discrepancy. The first is that Ćuk \& Gladman (2005) use a very simplified model for the tides acting on Triton, which does not have the same scaling with $q$ as our formulation. Their tidal damping time scale proceeds as proportional to $q^{6}$ while ours goes as $q^{15 / 2}$. Secondly, Ćuk \& Gladman (2005) average the tidal equation over one Kozai cycle. While from their description it is not clear how this is done, one would suspect that this results in using an averaged value of $q$ in the tidal equation rather than the minimum value. The latter controls the time scale for circularisation.

After its capture Triton undergoes at least another 3.6 Gyr of tidal evolution due to tides raised on Neptune by Triton. Even though the influence of tides raised on Neptune by Triton are a couple of orders of magnitude weaker than tides raised on Triton by Neptune, the subsequent evolution after Triton becomes circular cannot be ignored. For a circular orbit of Triton raising tides on Neptune we have

$$
\dot{a}=\frac{2 K_{N}}{m_{t} a_{T}}\left(\frac{\cos i_{T} \omega_{N}}{n_{T}}-1\right) .
$$

Since $\cos i_{T} \omega_{N} / n_{T} \gg 1$, we can ignore the second factor in the brackets, which makes the equation integrable with solution $a_{f}=\left(a_{i}^{13 / 2}+C t\right)^{2 / 13}$ where $C=6 k_{2 N} m_{T} R_{N}^{5} \Delta t_{N} \omega_{N} \cos i_{T} \sqrt{G / m_{N}}$. Note that for retrograde orbits $C<0$ and the orbit shrinks. Over 3.6 Gyr Triton's orbit should have shrunk by about $1.3 R_{N}$, from $15.6 R_{N}$ to its current value, making Triton's final position compatible with the tidal model at the $20 \%$ level or less.

Finally, Fig. 10 shows histograms of the distribution of the final inclination with respect to Neptune's equator of all circular orbits that used case 1 . There is a large surplus of retrograde objects and the majority of these are situated between $140^{\circ}$ and $150^{\circ}$, slightly lower than Triton's current value of $157^{\circ}$. The large number of objects in this bin can partially be 


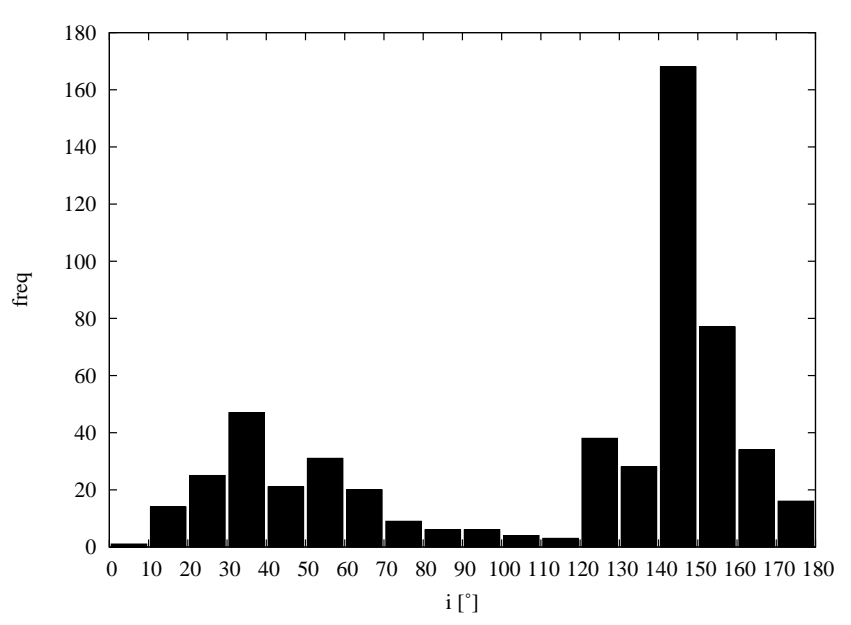

Figure 10: Histograms of the frequency of the final inclination with respect to Neptune's equator for case 1 i.e. with Neptune's current obliquity.

explained by the following. The inclination of Triton with respect to Neptune's equator $(i)$ is related to its inclination with respect to Neptune's orbit $(I)$, Neptune's obliquity $\left(\varepsilon_{N}\right)$ and the longitude of the ascending node $(\Omega)$ by $\cos i=\cos \varepsilon_{N} \cos I-$ $\sin \varepsilon_{N} \sin I \cos \Omega$. Since $\cos i$ is almost a constant for bodies close to Neptune, the value of $I$ varies between $i+\varepsilon_{N}$ and $i-\varepsilon_{N}$ with the circulation time of $\Omega$. Consequently, this same final value of $i$ can arise from orbits with original inclinations $I+\varepsilon_{N}$ and $I-\varepsilon_{N}$, where the final inclination depends on the original value of $\Omega$. The maximum value of $I$ is $180^{\circ}$ and so $180^{\circ}-\varepsilon_{N}=151.5^{\circ}$. Thus, the bin between $140^{\circ}$ and $150^{\circ}$ can sample orbits from the full range $\left(I+\varepsilon_{N}, I-\varepsilon_{N}\right)$, but orbits with higher $i$ cannot. Alternatively, orbits with lower values of $i$ sample orbits with $I<120^{\circ}$, where the Kozai mechanism operates strongly and can drive orbits to collide with Neptune. These collisions are the reason for the paucity of orbits with final $i \sim 90^{\circ}$, even if these orbits are protected from Kozai mechanism at small semi-major axis because of Neptune's $J_{2}$. Similarly, the maximum between $30^{\circ}$ and $40^{\circ}$ can be explained in a similar manner, though the number difference with the corresponding retrograde case is most likely a result of the retrograde cases being stable up to larger distances from Neptune.

We close this subsection by presenting some statistics for the case where Neptune's obliquity is not zero since this is the most probable. Of all the Tritons that we analysed, $3 \%$ are captured with an orbit having $q<1 R_{N}$, so that these collide with Neptune promptly after their capture. Of the remaining population there are three possible outcomes: circular with final semi-major axis $a>5 R_{N}$, eccentric orbit with final semi-major axis $a>5 R_{N}$, and tidal evolution to inside of $5 R_{N}$ before becoming circular. The last category consists of approximately $33 \%$ of the total population, so that some $67 \%$ survive on orbits with final semi-major axis $a>5 R_{N}$. This surviving population has only $29 \%$ on orbits with $e>10^{-5}$ or $19 \%$ of the total population. Combining these numbers it turns out that a circular Triton with final $a>5 R_{N}$ occurs approximately $50 \%$ of the time, with twice as many retrograde vs prograde cases. If Nep- tune's obliquity were zero, the above statistics are virtually the same.

\subsection{Probabilities}

Next we examine whether or not the existence of Triton is compatible with a capture during the planetary instability. Here we just concern ourselves with how many bodies of Triton's size are inferred to have existed from Triton's current orbit. However, before we continue we should pause for a moment and reflect on what we are interested in. The question is whether or not the capture of a Triton-like body is plausible, but not be so common that Uranus would have experienced the same event. We can make this argument even stronger: the Uranian satellite appears to be unperturbed and regular while Neptune's system appears to have been disrupted. For this to occur it is unimportant if the perturbing body was captured prograde or retrograde, and whether or not the orbit is circular at the current epoch. Only if the capture is a probable event is it worthy to ask the question about the most likely end states.

The probability of Triton's capture through the dissociation of a binary, and its subsequent evolution, is split into the product of two probabilities: the probability that over its whole lifetime a binary suffers an encounter with Neptune close enough for it to be disrupted $\left(P_{1}\right)$, and the probability that the binary member of Triton's mass is actually captured $\left(P_{2}\right)$. Their product is Triton's capture probability. We could restrict ourselves to only circular and retrograde outcomes, and thus we need to multiply the above total probability by the probability that after capture Triton reaches a circular orbit at the current epoch and is retrograde $\left(P_{3}\right)$. However being circular and retrograde at the current epoch is not necessary for disrupting Neptune's primordial satellite system.

The probability $P_{1}$ should reflect the encounter history of the binary until its distruption. Each encounter with Neptune softens the binary and, provided it is not disrupted, after many encounters it has no knowledge of its original binding energy (Parker \& Kaverlaars, 2010). However, we cannot take this history into account in our current methods but given that the probability of passing Neptune close enough to be disrupted is approximately $10^{-4}$ and that each planetesimal undergoes approximately 100 encounters, we believe that our method is accurate enough. It is not necessary to disrupt the binary on its first passage and thus we have used the total probability of disruption averaged over many encounters rather than just for the first encounter.

The value of $P_{1}$ depends on the distance from Neptune at which the binary gets disrupted, which in turn depends on the mass ratio and total mass of the binary. Similarly, $P_{2}$ depends on the system configuration to determine how many Tritons are captured. In table 4 we list the values of $P_{1}$ and $P_{2}$ for the binary systems that we considered, in units of percent. The values of $P_{1}$ are for encounters that occur during and after the planetary instability, since we have information about how much mass there was available at this time (Gomes et al., 2005). Since the 


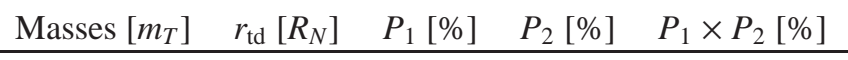

$\begin{array}{llllc}1.0,0.1 & 35 & 3.9 & 19.3 & 0.75 \\ 1.0,0.3 & 33 & 3.7 & 19.8 & 0.73 \\ 1.0,1.0 & 29 & 3.1 & 36.9 & 1.1 \\ 3.0,1.0 & 23 & 2.1 & 11.8 & 0.25\end{array}$

Table 4: Table with probabilities $P_{1}$ and $P_{2}$ and the total capture probability $P_{c}=P_{1} \times P_{2}$ for the various binary systems that we considered.

\begin{tabular}{ccc} 
Case & $P_{3}[\%]$ & $P[\%]$ \\
\hline 1 & 30.1 & 0.21 \\
2 & 29.8 & 0.21
\end{tabular}

Table 5: Probabilities $P_{3}$ and the total combined probability of capture and a circular retrograde orbit at the current epoch, $P=P_{1} \times P_{2} \times P_{3}$, for tidal cases 1 and 2.

cumulative distribution of the binary's peri-Neptune distance is linear, the probability of disruption $P_{1} \propto r_{t d} \propto\left(m_{1}+m_{2}\right)^{-1 / 3}$, which is the approximate trend observed in the table.

The value of $P_{3}$ depends on the tidal models that we used. Its values are listed in Table 5 and the unit is again percent. Both cases 1 and 2 are considered. The value of $P=P_{1} \times P_{2} \times P_{3}$ is also listed, where we used the average values of $P_{1}$ and $P_{2}$ from the previous table.

The total probability of a successful capture, $P_{c}=P_{1} \times P_{2}$, is on average approximately 1:140. If we then restrict ourselves to Triton being retrograde and circular at the current epoch, the probability becomes $P=1: 500$. We can use these values to constrain the number of primordial binaries, $N_{B}$, having one Tritonsized member. The probability of having at least one Triton capture if we have $N_{B}$ Triton binaries is 1 minus the probability of its negation, that is, the probability of having no capture after all $N_{B}$ cases. Since the events are independent this total probability becomes the product of each individual probability of not having a capture. Each individual probability of not having a capture is $1-P=139 / 140$ so that the probability of having no capture after $N_{B}$ trials is $(139 / 140)^{N_{B}}$. The probability of having at least one capture is of course its complement $P_{\text {tot }}=1-(1-P)_{B}^{N}=1-(139 / 140)^{N_{B}}$. Since Neptune's system appears to be disrupted and the Uranian system appears to be regular, the maximum value of $P_{\text {tot }}$ is approximately $50 \%$. Solving for $N_{B}$ we obtain $N_{B}=97$ for the number of binaries at the planetary instability epoch with at least one Triton-sized member. Is this consistent with current theory? Morbidelli et al. (2009) claim there were $N_{T} \sim 1000$ Tritons in the transNeptunian disc at the planetary instability epoch, within factor of a few. This implies a primordial binary population with at least one Triton-sized member, and with the mass ratios that we considered, of approximately $10 \%$. The primordial Kuiper Belt population is thought to have consisted of 5\%-40\% binaries (Burns, 2004; Noll et al., 2008; Lin et al., 2010), so that our value of $N_{B}$ is consistent with this estimate, though only at the lowest level. However, the above fraction corresponds to smaller size bodies than the ones we are interested in here, and there is some indirect evidence that the primordial binary population for heavier bodies is lower (Brown et al., 2006). Thus we conclude that the Agnor \& Hamilton (2006) binary capture scenario during the planetary instability is consistent with Triton's existence.

However, in the above argument we did not place any restriction on the final semi-major axis, eccentricity and inclination of Triton. Placing restraints on the final semi-major axis and inclination will decrease the final probability, $P$, because a fourth probability, $P_{4}$, comes into play. It would contain information about the chances of having Triton end up in a specific range of semi-major axis and/or inclination. This introduces the question of choosing a suitable bin size for the final semi-major axis and/or inclination that could be compatible with Triton's current orbit. We prefer not to do that here and instead only quote the probability of Neptune ending up with a circular, retrograde satellite of Triton's mass.

Given that Neptune has a large, retrograde satellite and the other giant planets do not, a natural question to ask is why did this not happen for the other giant planets. The only reason we can think of is that Neptune encounters more planetesimals than the other giant planets (see table 1). Even though Uranus encounters a similar number, the encounters occur at a greater speed which decreases the probability of capture.

\section{Nereid: The fly in the ointment}

In the previous section we have presented the results of our numerical simulations. We concluded that the capture of Triton through the dissociation of a binary that had a deep encounter with Neptune, followed by subsequent tidal evolution that left Triton semi-molten (McKinnon, 1984), is enough to place Triton on its current circular, retrograde orbit. No extra ingredients, such as the collision with a hypothetical regular satellite of Neptune (Goldreich et al., 1989), the presence of a gas disc (McKinnon \& Leith, 1995) or a debris disc left over from the mutual collisions among the members of a fictitious regular satellite system (Ćuk \& Gladman, 2005) are needed (Correia, 2009). Does that mean that we are done?

No, because there are several issues that we did not address. The first of these is what would have happened to Neptune's irregular satellites if Triton were captured during the planetary instability, when the other irregular satellites were captured too (Nesvorný et al., 2007). While Triton remained on a highlyeccentric orbit, with a semi-major axis comparable to that of the other irregular satellites, it greatly perturbs the rest of this population (Ćuk \& Gladman, 2005). Nereid in particular is difficult to keep, and Ćuk \& Gladman (2005) conclude that it will be lost within 0.1 Myr. However, the initial conditions of Ćuk \& Gladman (2005) are somewhat artificial and direct comparison with our post-capture orbits is difficult. Therefore we have performed similar simulations, in which we place Triton on an 

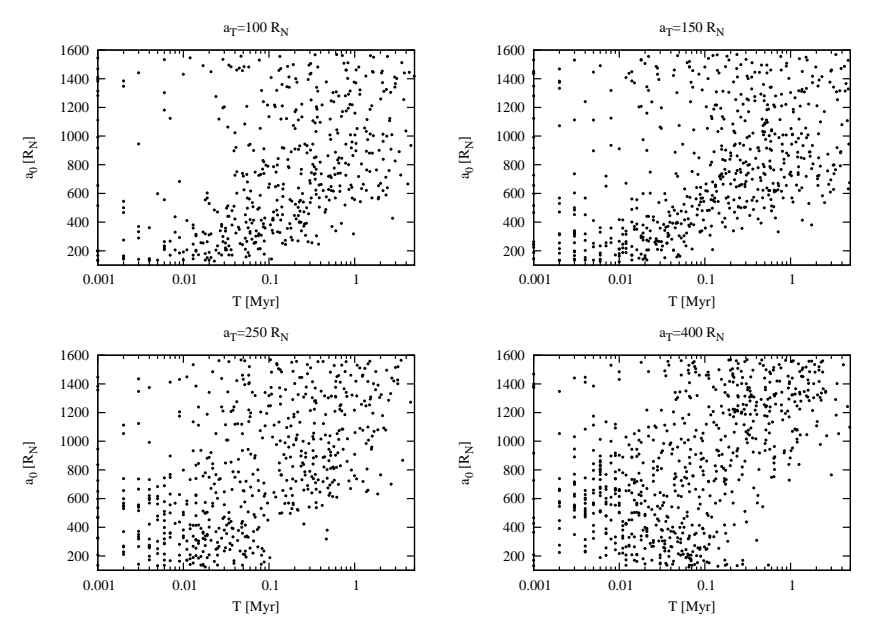

Figure 11: Lifetime vs. initial semi-major axis of a fictitious swarm of irregular satellites of Neptune that were perturbed by Triton. The initial pericentre of Triton was set at $7 R_{N}$ but the semi-major axis was varied (see titles above panels for values).

eccentric orbit with semi-major axis ranging from 100 to 400 Neptune radii, pericentre distance $q \sim 7 R_{N}$ and investigated how its presence affected Nereid and a swarm of other irregular satellites. We placed 1000 fictitious irregular satellites around Neptune where the initial conditions were taken from Nesvorný et al. (2007). The Sun was added as an external perturber. It turned out that Nereid is always lost through collision with Neptune within $0.1 \mathrm{Myr}$, even if it was captured with an initially almost circular orbit. The longest stability was found when Triton's semi-major axis was less than $100 R_{N}$. Using the tidal model, Triton needs of the order of $\sim 100$ Myr to become circular. The eccentric phase lasts for approximately $T_{a}=a / \dot{a}$ which for highly-eccentric orbits becomes

$$
T_{a}=1.8\left(\frac{q}{7 R_{N}}\right)^{15 / 2}\left(\frac{a}{100 R_{N}}\right)^{1 / 2} \quad \text { Myr. }
$$

Thus when Triton's semi-major axis is $200 R_{N}$ and $q \sim 7 R_{N}$ it stays eccentric for approximately $3 \mathrm{Myr}$, but if $a=100 R_{N}$ it is not even 2 Myr. This simple approximation does not take Kozai mechanism into account, which increases the time by approximately one order of magnitude. Thus, with this model Triton stays eccentric for far too long and we lose Nereid. We have presented the results of some numerical simulations in Fig. 11. The panels plot the time a satellite is lost vs. its initial semimajor axis. As can be seen, even when Triton has an orbit with just $a=100 R_{N}$, Nereid (at $a=222 R_{N}$ ) is lost within $0.1 \mathrm{Myr}$, even when Nereid's initial eccentricity is close to zero. Finally, Fig. 12 shows the original semi-major axis and eccentricity of the irregular satellites that are lost (bullets) and that survive (open circles). The big filled squares indicate Neptune's current irregular satellites Nereid, Halimede, Sao and Laomedeia. The farthest two, Psamathe and Neso, are off the scale to the right. As one can see, Nereid is always lost, even if it was captured with a low eccentricity. Only satellites captured with a low eccentricity and which also have a semi-major axis $a \gtrsim 2 a_{T}$ survive.
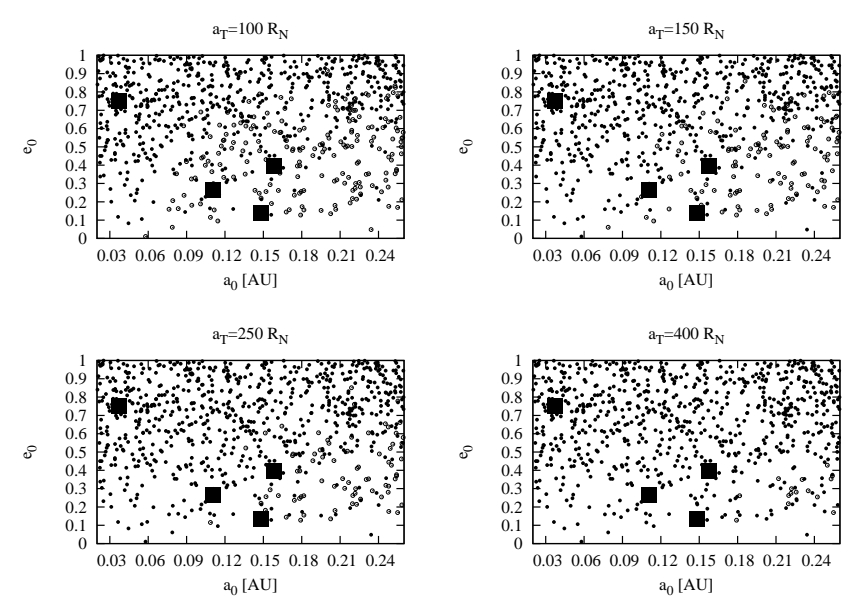

Figure 12: Original semi-major axis vs. initial eccentricity of a fictitious swarm of irregular satellites of Neptune that were perturbed by Triton. Bullets show objects that are lost, while the open circles show objects that survive. Large filled squares show the positions of Neptune's currently-known irregular satellites Nereid, Halimede, Sao and Laomedeia. The initial pericentre of Triton was set at $7 R_{N}$ but the semi-major axis was varied (see titles above panels for values).

There are five ways out of this dilemma that we can think of. The first is that Triton could have been captured with a very small semi-major axis, smaller than $\sim 50 R_{N}$. In the top panel of Fig. 13 we plotted the cumulative distribution of the original semi-major axis upon capture for all objects which reached an eccentricity $e=10^{-5}$ within the age of the solar system. The solid line is for case 1 , the dashed line represents case 2 . As one can see, only $5 \%$ of the time is Triton captured with a semi-major axis $\lesssim 50 R_{N}$. The median value is around $200 R_{N}$, approximately where Nereid is $\left(a=222 R_{N}\right)$, which is much lower than the median value upon capture (Fig. 5). Thus, capture at small semi-major axis is unlikely, but not impossible. For reference, the bottom panel contains the cumulative semimajor axis distribution of objects with $a>5 R_{N}$ and $e>10^{-5}$. Most of these are still exhibiting Kozai oscillations and have undergone very little tidal evolution.

A second possible exit strategy is to argue that Nereid was not captured by the mechanism of Nesvorný et al. (2007) and instead was a regular satellite of Neptune that was scattered outwards by Triton. We performed a series of numerical simulations to test this hypothesis. We placed a large number of test particles on circular, equatorial orbits around Neptune with a maximum semi-major axis of $45 R_{N}$, which is twice as far as Oberon is from Uranus. We have not witnessed any regular satellite being scattered to Nereid's orbit by Triton. Instead, they all collide with Neptune within a few tens of thousands of years, or they are ejected by Triton when their orbits reach $q \lesssim 3 R_{N}$ and their orbital angular momentum is at a minimum. Even if Triton could place a regular satellite on Nereid's orbit, we are still faced with the dilemma of keeping it there afterwards. In addition, we have one additional argument against the scattering scenario. 

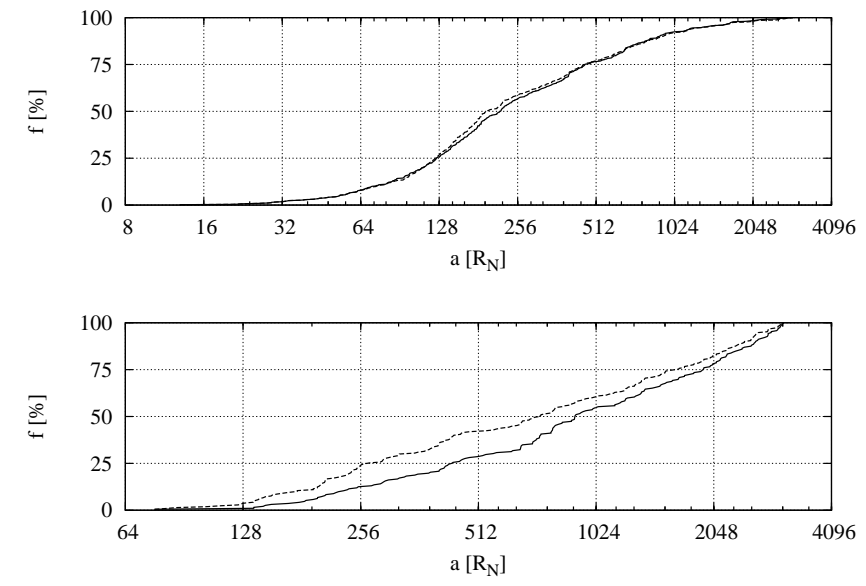

Figure 13: Top panel: Cumulative distribution of semi-major axis upon capture of objects that reach eccentricity $e=10^{-5}$ within the age of the solar system. The solid line represents case 1 , the dashed line is case 2. Bottom panel: the same as the top panel but for orbits with final $a>5 R_{N}$ and $e>10^{-5}$.

The measured rotation period of Nereid is $13.6 \mathrm{~h}$ (Grav et al., 2003). This has two implications: i) either Nereid was captured on its current orbit and thus the rotation period that we see is a remnant from when it formed, because it is too far away from Neptune to be tidally despun, or ii) it used to be on a $13.6 \mathrm{~h}$ orbit around Neptune and was scattered to its current orbit by Triton. A period of $13.6 \mathrm{~h}$ corresponds to a semi-major axis of approximately $3 R_{N}$, closer in than Proteus at $4.7 R_{N}$. If Nereid was any further from Neptune its rotation period would be longer than it is now. So it is extremely unlikely that Triton scattered a synchonous Nereid from close to Neptune to its current orbit without messing up the rest of the system, in particular the satellites Proteus and Galatea. Thus a captured origin for Nereid is the most plausible. If this capture occured around the same time as that of Triton, Nereid would be lost through collision with Neptune.

A third scenario has been proposed by Desch \& Porter (2010), who investigated the idea of Triton having been a satellite of a 2 Earth-mass fictitious planet called Amphitrite. When the binary Amphitrite-Triton suffered a close approach with Neptune, Triton was captured in orbit around Neptune. Desch \& Porter (2010) speculated that Amphitrite could later collide with Uranus to produce its axial tilt or with Neptune itself to account for its excess heat radiation. Assuming that Amphitrite collided with Neptune and that this caused the capture of Triton, Desch \& Porter (2010) state the probability of capturing Triton in this manner is approxiately $20 \%-40 \%$ for encounter velocities at Neptune's Hill sphere of less than $3 \mathrm{~km} \mathrm{~s}^{-1}$. Their typical pericentre distance of Triton after captre is $7 R_{N}$, for original Amphitrite-Triton semi-major axis shorter than $40 R_{N}$. Unfortunately Desch \& Porter (2010) do not give any information about the typical semi-major axis of Triton after capture, so that it is unclear if this capture mechanism is able to capture Triton at a short-enough semi-major axis in order to prevent the loss of Nereid. In addition, for low encounter velocities and long initial semi-major axis of the binary, the pericentre distance of Triton upon capture is large and tides may not be able to circularise it within the age of the Solar System.

A fourth scenario involves the debris disc proposed by Ćuk \& Gladman (2005). However, as we stated in the introduction, it is not clear whether or not Neptune's hypothetical regular satellites will grind themselves down to a debris disc before one of them collides with Triton and shatters both Triton and itself. In principle the satellites collide with each other because Triton forces their eccentricities. This forcing is inversely proportional to the semi-major axis ratio of the regular satellites and Triton, and Triton's eccentricity. Furthermore, if Triton's semi-major axis is very long it is energetically more favourable for the satellites to eject Triton. Thus we envision three possible outcomes as a function of increasing original semi-major axis of Triton after capture. When Triton's semi-major axis is short, mutual collision among the satellites, which form the debris disc, is the most likely outcome because Triton induces a large eccentricity in the satellites. For intermediate semi-major axis of Triton, the forced eccentricities of the satellites are not large enough for them to cross each other and Triton will hit one of these satellites before it is ejected. For very long semi-major axes of Triton, it will be ejected before a collision occurs. We reserve investigating this scenario for future work.

The fifth, most plausible, scenario is that Triton was captured and circularised before the planetary instability, something which was already suggested by Vokrouhlický et al. (2009) but for different reasons. This early capture scenario would solve the problem of destabilising Neptune's other irregular satellites, such as Nereid, because it was unlikely that they were already there. Since Neptune might not have had a circumplanetary gas disc but just a cooling, rotating envelope (Ayliffe \& Bate, 2009), the perturbations from Triton could have disturbed this system enough to prevent the formation of a regular satellite system such as that of Uranus, while decreasing its orbital energy at the same time. Proteus' current position would be indicative of the minimum distance Triton reached after its capture prior to reaching its current orbit. In addition, at this early stage Neptune's obliquity may have been close to 0 , increasing the likelihood of Triton ending up on its current orbit (see Fig. 8). Neptune's low obliquity would also have changed the final inclination distribution, which we have plotted in Fig. 14 As one can see, the retrograde orbits are more evenly spread over a larger interval. We should add that it is possible that the final semi-major axis and inclination distribution of captured objects before the planetary instability would be different than that presented above, because the binary encounters with Neptune would have occurred at different velocities since Neptune was most likely closer to the Sun, and because it was not migrating. However, we do not think that the final results would be qualitatively very different from what has been presented above, although the probabilities discussed earlier would most likely change. The early capture scenario also revokes the need for a debris disc resulting from mutual collisions among fictitious regular satellites of Neptune (Ćuk \& Gladman, 2005) or a col- 


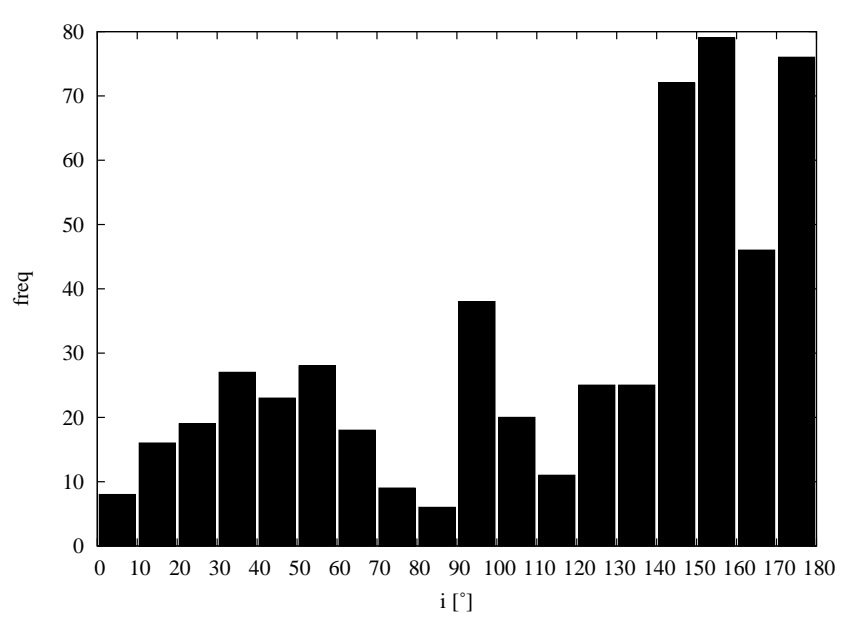

Figure 14: Histograms of the frequency of the final inclination with respect to Neptune's equator for case 2 i.e. without Neptune's obliquity.

lision with such a fictitious satellite (Goldreich et al, 1989) and only requires the minimum ingredients: a binary capture and tidal evolution.

\section{Summary and conclusions}

We have made an attempt at determining the origin of Triton, Neptune's large, circular, retrograde satellite. Our work is based on two assumptions. The first is that Triton was captured through a binary exchange (Agnor \& Hamilton, 2006) and that the subsequent shrinking and circularisation of the orbit occurred through tides only (Correia, 2009). We performed numerical simulations of the migration of Neptune in the framework of the Nice model (Tsiganis et al., 2005) and recorded the close encounters that the planetesimals suffered with Neptune. The closest of these encounters were re-enacted with binaries of various mass ratios to determine whether the capture of Triton would occur, and for what parameters. The re-enaction experiments yielded a set of initial orbits for Triton just after its capture. These post-capture orbits were integrated using the tidal model of Mignard (1979, 1980) and Hut (1981), as was done by Correia (2009). However, we added the effects of Kozai mechanism induced by the Sun (Kozai, 1962) and the effect of Neptune's figure (e.g. Kinoshita \& Nakai, 1991). The former will cause oscillations in Triton's eccentricity and inclination. Thus Triton's orbital angular momentum, which is constant when only tides are taken into account, is no longer conserved. The perturbations from the figure of Neptune will overrule the Kozai effect once Triton is close enough to Neptune, so that the eccentricity and inclination oscillations cease. We integrated the tidal equations until Triton's orbit was circu$\operatorname{lar}\left(e<10^{-5}\right)$.

We find that a binary capture and tidal evolution are sufficient to reproduce Triton's current orbit, even though the tidal model would predict Triton to be closer to Neptune than its current position at $14.3 R_{N}$. The probability of Neptune having a
Triton-mass satellite is $0.7 \%$. From this we deduce there were $\sim 100$ binaries in the trans-Neptunian disc with at least one Triton-sized member. This number is consistent with theoretical predictions, though at the low end.

The typical time for a final orbit at $14 R_{N}$ to become circular is of the order of $200 \mathrm{Myr}$, much shorter than the age of the solar system or the time between the formation of the giant planets and the planetary instability. However, the Kozai oscillations caused by the perturbations from the Sun increase the time that Triton stays in an eccentric orbit. The perturbations from an eccentric Triton destabilise Nereid on a time scale of less than $0.1 \mathrm{Myr}$, so that its existence is in contradiction with our model. The most plausible exit strategies are either capture of Triton at small semi-major axis (a rare event), or a capture before the planetary instability when Nereid was not there. The early capture of Triton would remove the need for a fast circularisation of its orbit, decrease the possibility of a collision with an existing regular satellite and also increase the probability of Triton ending up on its current orbit because Neptune's obliquity may have been close to zero. Thus, we suggest that Triton was captured shortly after Neptune's formation through a binary encounter and was circularised to its current orbit through tides, while possibly disturbing Neptune's rotating envelope (Ayliffe $\&$ Bate, 2009) and preventing the formation of other regular satellites.

\section{Acknowledgements}

The authors wish to express their gratitude to Alexandre Correia for making available to us some of his data and for many helpful discussions. RB sincerely thanks Germany's Helmholtz Alliance through their 'Planetary Evolution and Life' programme for financial support. RG and EN express their gratitude towards Brazil's CNPq and FAPERJ for their funding. Part of this work was accomplished while RB visited ON/MCT and he thanks his hosts RG and EN for their hospitality and Brazil's CNPq for financing his trip.

\section{Bibliography}

Agnor, C., Asphaug, E. 2004. Accretion Efficiency during Planetary Collisions. The Astrophysical Journal 613, L157-L160.

Agnor, C. B., Hamilton, D. P. 2006. Neptune's capture of its moon Triton in a binary-planet gravitational encounter. Nature 441, 192-194.

Anderson, J. D., Jacobson, R. A., Lau, E. L., Moore, W. B., Schubert, G. 2001. Io's gravity field and interior structure. Journal of Geophysical Research 106, 32963-32970.

Ayliffe, B. A., Bate, M. R. 2009. Circumplanetary disc properties obtained from radiation hydrodynamical simulations of gas accretion by protoplanets. Monthly Notices of the Royal Astronomical Society 397, 657-665.

Benner, L. A. M., McKinnon, W. B. 1995. Orbital behavior of captured satellites: The effect of solar gravity on Triton's postcapture orbit.. Icarus 114, 1-20. Bernstein, G. M., Trilling, D. E., Allen, R. L., Brown, M. E., Holman, M., Malhotra, R. 2004. The Size Distribution of Trans-Neptunian Bodies. The Astronomical Journal 128, 1364-1390.

Brown, M. E., van Dam, M. A., Bouchez, A. H., Le Mignant, D., Campbell, R. D., Chin, J. C. Y., Conrad, A., Hartman, S. K., Johansson, E. M., Lafon, R. E., Rabinowitz, D. L., Stomski, P. J., Jr., Summers, D. M., Trujillo, C. A., 
Wizinowich, P. L. 2006. Satellites of the Largest Kuiper Belt Objects. The Astrophysical Journal 639, L43-L46.

Bulirsch, R., Stoer, J. 1966. Numerical treatment of ordinary differential equations by extrapolation methods. Numerische Mathematik 8, 1-13.

Burns, J. A. 2004. Planetary science: Double trouble. Nature 427, 494-495.

Canup, R. M., Ward, W. R. 2006. A common mass scaling for satellite systems of gaseous planets. Nature 441, 834-839.

Chambers, J. E. 1999. A hybrid symplectic integrator that permits close encounters between massive bodies. Monthly Notices of the Royal Astronomical Society 304, 793-799.

Correia, A. C. M. 2009. Secular Evolution of a Satellite by Tidal Effect: Application to Triton. The Astrophysical Journal 704, L1-L4.

Ćuk, M., Gladman, B. J. 2005. Constraints on the Orbital Evolution of Triton. The Astrophysical Journal 626, L113-L116.

Desch, S., Porter, S. 2010. Amphitrite: A Twist on Triton's Capture. Lunar and Planetary Institute Science Conference Abstracts 41, 2625.

Duncan, M., Quinn, T., Tremaine, S. 1987. The formation and extent of the solar system comet cloud. The Astronomical Journal 94, 1330-1338.

Efroimsky, M., Lainey, V. 2007. Physics of bodily tides in terrestrial planets and the appropriate scales of dynamical evolution. Journal of Geophysical Research (Planets) 112, 12003.

Fernández, J. A. 1981. New and evolved comets in the solar system. Astronomy and Astrophysics 96, 26-35.

Fernández, J. A. 1997. The Formation of the Oort Cloud and the Primitive Galactic Environment. Icarus 129, 106-119.

Goldreich, P., Murray, N., Longaretti, P. Y., Banfield, D. 1989. Neptune's story. Science 245, 500-504.

Gomes, R. S., Morbidelli, A., Levison, H. F. 2004. Planetary migration in a planetesimal disk: why did Neptune stop at 30 AU? Icarus 170, 492-507.

Gomes, R., Levison, H. F., Tsiganis, K., Morbidelli, A. 2005. Origin of the cataclysmic Late Heavy Bombardment period of the terrestrial planets. Nature 435, 466-469.

Grav, T., Holman, M. J., Kavelaars, J. J. 2003. The Short Rotation Period of Nereid. The Astrophysical Journal 591, L71-L74.

Hamilton, D. P., Krivov, A. V. 1997. Dynamics of Distant Moons of Asteroids. Icarus 128, 241-249.

Hut, P. 1981. Tidal evolution in close binary systems. Astronomy and Astrophysics 99, 126-140.

Jacobson, R. A., Riedel, J. E., Taylor, A. H. 1991. The orbits of Triton and Nereid from spacecraft and earthbased observations. Astronomy and Astrophysics $247,565-575$.

Jacobson, R. A., Campbell, J. K., Taylor, A. H., Synnott, S. P. 1992. The masses of Uranus and its major satellites from Voyager tracking data and earth-based Uranian satellite data. The Astronomical Journal 103, 2068-2078.

Karato, S.-I. 2008. Deformation of Earth Materials: An Introduction to the Rheology of Solid Earth. Cambridge Univ. Press, Cambridge, UK

Kinoshita, H., Nakai, H. 1991. Secular perturbations of fictitious satellites of Uranus. Celestial Mechanics and Dynamical Astronomy 52, 293-303.

Kinoshita, H., Nakai, H. 2007. General solution of the Kozai mechanism. Celestial Mechanics and Dynamical Astronomy 98, 67-74.

Kozai, Y. 1962. Secular perturbations of asteroids with high inclination and eccentricity. The Astronomical Journal 67, 591-598.

Lainey, V., Dehant, V., Pätzold, M. 2007. First numerical ephemerides of the Martian moons. Astronomy and Astrophysics 465, 1075-1084.

Lainey, V., Arlot, J.-E., Karatekin, Ö., van Hoolst, T. 2009. Strong tidal dissipation in Io and Jupiter from astrometric observations. Nature 459, 957-959.

Levison, H. F., Morbidelli, A., Vanlaerhoven, C., Gomes, R., Tsiganis, K. 2008.
Origin of the structure of the Kuiper belt during a dynamical instability in the orbits of Uranus and Neptune. Icarus 196, 258-273.

Levison, H. F., Bottke, W. F., Gounelle, M., Morbidelli, A., Nesvorný, D., Tsiganis, K. 2009. Contamination of the asteroid belt by primordial trans-Neptunian objects. Nature 460, 364-366.

Lin, H.-W., Kavelaars, J. J., Ip, W.-H., Gladman, B. J., Petit, J. M., Jones, R. L., Parker, J. W. 2010. On the Detection of Two New Trans-Neptunian Binaries from the CFEPS Kuiper Belt Survey. Publications of the Astronomical Society of the Pacific 122, 1030-1034.

MacDonald, G. J. F. 1964. Tidal Friction. Reviews of Geophysics and Space Physics 2, 467-541.

Marty, J. C., Balmino, G., Duron, J., Rosenblatt, P., Le Maistre, S., Rivoldini, A., Dehant, V., van Hoolst, T. 2009. Martian gravity field model and its time variations from MGS and Odyssey data. Planetary and Space Science 57, 350363.

McCord, T. B. 1966. Dynamical evolution of the Neptunian system. The Astronomical Journal 71, 585.

McKinnon, W. B. 1984. On the origin of Triton and Pluto. Nature 311, 355-358. McKinnon, W. B., Leith, A. C. 1995. Gas drag and the orbital evolution of a captured Triton.. Icarus 118, 392-413.

Mignard, F. 1979. The evolution of the lunar orbit revisited. I. Moon and Planets 20, 301-315.

Mignard, F. 1980. The evolution of the lunar orbit revisited. II. Moon and Planets 23, 185-201.

Morbidelli, A., Levison, H. F., Tsiganis, K., Gomes, R. 2005. Chaotic capture of Jupiter's Trojan asteroids in the early Solar System. Nature 435, 462-465.

Morbidelli, A., Tsiganis, K., Crida, A., Levison, H. F., Gomes, R. 2007. Dynamics of the Giant Planets of the Solar System in the Gaseous Protoplanetary Disk and Their Relationship to the Current Orbital Architecture. The Astronomical Journal 134, 1790-1798.

Morbidelli, A., Levison, H. F., Bottke, W. F., Dones, L., Nesvorný, D. 2009. Considerations on the magnitude distributions of the Kuiper belt and of the Jupiter Trojans. Icarus 202, 310-315.

Nesvorný, D., Vokrouhlický, D., Morbidelli, A. 2007. Capture of Irregular Satellites during Planetary Encounters. The Astronomical Journal 133, 19621976.

Noll, K. S., Grundy, W. M., Chiang, E. I., Margot, J.-L., Kern, S. D. 2008. Binaries in the Kuiper Belt. The Solar System Beyond Neptune. 345-363. M. A. Barucchi, H. Boehnhardt, D. P. Cruikshank, A. Morbidelli (eds). University of Arizona Press, Tucson, AZ, USA.

Öpik, E. 1976. Interplanetary Encounters. Elsevier Scientific Publishing, Amsterdam.

Parker, A. H., Kavelaars, J. J. 2010. Destruction of Binary Minor Planets During Neptune Scattering. The Astrophysical Journal 722, L204-L208.

Tsiganis, K., Gomes, R., Morbidelli, A., Levison, H. F. 2005. Origin of the orbital architecture of the giant planets of the Solar System. Nature 435, 459-461. Vokrouhlický, D., Nesvorný, D., Levison, H. F. 2008. Irregular Satellite Capture by Exchange Reactions. The Astronomical Journal 136, 1463-1476.

Ward, W. R., Canup, R. M. 2003. Viscous Evolution of an Impact Generated Water/Rock Disk Around Uranus. Bulletin of the American Astronomical Society 35, 1046.

Williams, J. G., Boggs, D. H., Ratcliff, J. T. 2005. Lunar Fluid Core and Solid Body Tides, LPI 36, 1503.

Yoder, C. F., Konopliv, A. S., Yuan, D. N., Standish, E. M., Folkner, W. M. 2003. Fluid Core Size of Mars from Detection of the Solar Tide. Science 300, 299-303. 